\title{
TRABES ACARTELADAS DE CONCRETO REFORZADO CONTINUAS DISEÑADAS PARA FALLAR POR CORTANTE. PARTE 1: DESCRIPCIÓN DE LOS EXPERIMENTOS Y DEL COMPORTAMIENTO CÍCLICO
}

\author{
Arturo Tena Colunga ${ }^{(1)}$, Luis Angel Urbina Californias ${ }^{(2)}$ y Hans I. Archundia Aranda ${ }^{(3)}$
}

\begin{abstract}
RESUMEN
Se presentan resultados e interpretaciones de un programa experimental donde se han ensayando trabes acarteladas de concreto reforzado en condiciones de continuidad y sujetas a cargas cíclicas reversibles que permita estudiar su comportamiento a cortante. En este trabajo se reportan las características de los experimentos y la interpretación de los resultados de las primeras cuatro trabes acarteladas y una prismática de concreto reforzado que fueron diseñadas para fallar a cortante cuando disponen de refuerzo por cortante en la zona de las cartelas. Todos los especímenes tienen una longitud de acartelamiento de un tercio del claro libre y cubren uniformemente ángulos de acartelamiento entre cero (prismática) y diez grados. Los especímenes fueron ensayados a través de un patrón de cargas cíclicas reversibles y crecientes controladas por desplazamiento, donde se sometieron a dos ciclos completos a la misma amplitud de desplazamiento, aumentando la amplitud de los ciclos de desplazamientos conforme a un patrón geométrico establecido. Se discute el comportamiento cíclico de las trabes acarteladas en cuanto a sus patrones de agrietamiento, degradación de rigidez y resistencia y disipación de energía, y se compara con respecto a la de la trabe prismática de referencia. Los resultados de los ensayes en condiciones de continuidad permiten corroborar lo observado en los ensayes anteriores en condiciones de apoyo simple: que las trabes acarteladas de concreto reforzado son más eficientes que las trabes prismáticas, aún cuando fallan por cortante.
\end{abstract}

Palabras clave: trabes acarteladas; patrones de agrietamiento; capacidad de deformación; degradación de rigidez; disipación de energía

Artículo recibido el 29 de julio de 2016 y aprobado para su publicación el 2 de octubre de 2017. Se aceptarán comentarios y/o discusiones hasta cinco meses después de su publicación.

(1) Profesor, Departamento de Materiales, Universidad Autónoma Metropolitana Azcapotzalco, Av. San Pablo 180, Col. Reynosa Tamaulipas, 02200 México, DF, e-mail: $\underline{\text { atc@ correo.azc.uam.mx }}$

(2) Profesor, Universidad Politécnica de Chimalhuacán, Emiliano Zapata S/N, Col. Transportistas, 56363 Chimalhuacán, Edo. México, e-mail: ucla_1985@yahoo.com

(3) Profesor Departamento de Materiales, Universidad Autónoma Metropolitana Azcapotzalco, Av. San Pablo 180, Col. Reynosa Tamaulipas, 02200 México, DF, e-mail: $\underline{\text { archundia@azc.uam.mx }}$ 


\title{
CONTINUOUS REINFORCED CONCRETE HAUNCHED BEAMS DESIGNED TO FAIL IN SHEAR. PART 1: DESCRIPTION OF TEST SPECIMENS AND CYCLIC BEHAVIOR
}

\begin{abstract}
Research results and interpretations of the testing of five prototype continuous reinforced concrete beams (four haunched and one prismatic) designed to develop a shear failure under cyclic loading are presented. Subject beams were tested with minimum shear reinforcement. The studied haunched length is one-third the effective span of the beam. The considered angles of slope of haunch from horizontal vary from $0^{0}$ (prismatic) to $10^{\circ}$. Cyclic tests were displacement-controlled, and two cycles at the same displacement were set in the displacement history which considers a geometrical increment of target displacements. Differences in the cyclic shear behavior of haunched beams with respect to prismatic beams were monitored in terms of cracking patterns, stiffness and strength degradation and energy dissipation. The obtained results from the cyclic testing in continuity conditions allow one to corroborate what it was observed in previous testing for simply supported beams: reinforced concrete haunched beams are more efficient than reinforced concrete prismatic beams, even when they fail in shear.
\end{abstract}

Keywords: haunched beams; cracking patterns; deformation capacity; stiffness degradation; energy dissipation

\section{INTRODUCCIÓN}

Las trabes acarteladas de concreto reforzado son atractivas como alternativa estructural para edificios de dimensiones importantes y para puentes, porque conceptualmente proporcionan algunas ventajas sobre trabes prismáticas de concreto reforzado, como se detalla y enlista en trabajos previos (por ejemplo, Tena 1993, Tena-Colunga 1994, Tena-Colunga 1996, Archundia et al. 2007, Tena-Colunga et al. 2008). La mayor desventaja de estos elementos estructurales es que su cimbrado y armado son bastante más laboriosos que el de las trabes prismáticas, lo cual redunda en un mayor costo por este concepto.

El uso de trabes acarteladas en edificios y puentes a nivel mundial no es nuevo. Sin embargo, por razones económicas relacionadas con el costo de la mano de obra, su uso en países desarrollados es más común en puentes de todo tipo, mientras que en edificios se utilizan principalmente en México (figura 1a) y en otros países latinoamericanos, como Ecuador, donde la mano de obra es relativamente barata, aunque también se emplean de manera frecuente en puentes en México (figura 1b).

A pesar de ello, son escasas las investigaciones experimentales sobre el comportamiento de trabes acarteladas de concreto reforzado, y prácticamente todas se han limitado a estudiar su mecanismo resistente a cortante. La gran mayoría de los experimentos se han hecho en trabes simplemente apoyadas ante carga puntual estática monótona creciente (Mörsch 1952, Debaiky y El-Niema 1982, Stefanou 1983, El-Niema 1988, Archundia 2004, Archundia et al. 2005 y 2006, Tena-Colunga et al. 2008, Nghiep 2011, Hou et al. 2015). A continuación se sintetizan algunos de los trabajos pioneros en el tema. En Archundia (2013) se presenta una discusión analítica y exhaustiva de cada trabajo experimental citado líneas arriba. 


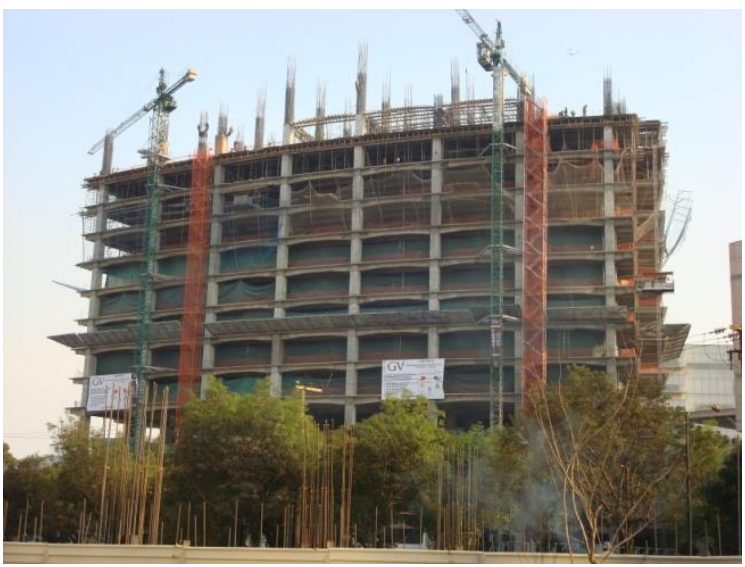

a) Av. Miguel de Cervantes

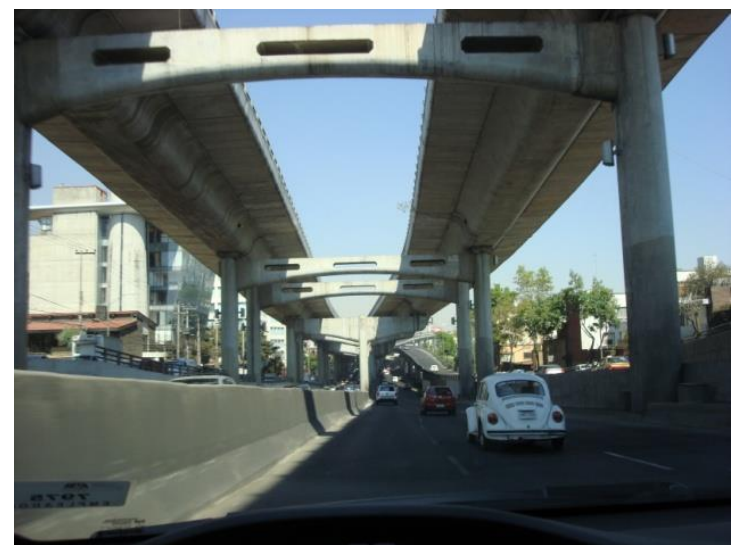

b) Periférico Sur

Figura 1. Puentes y edificios con trabes acarteladas en Ciudad de México

Los trabajos reportados por Mörsch (1952) son pioneros en este tema. Los primeros experimentos fueron hechos por Mörsch, en trabes acarteladas simplemente apoyadas de sección rectangular, donde la cartela se encontraba sujeta a compresión. De hecho, fue Mörsch quien en 1908 derivó las primeras ecuaciones de diseño por cortante para trabes de sección variable, además de dar recomendaciones prácticas para el detallado de las cartelas, entre muchos otros aspectos (Mörsch 1952, Archundia 2013).

Deibaiky y El-Niema (1982) ensayaron 33 especímenes simplemente apoyados de sección rectangular que sometieron a dos cargas simétricas en la sección de los extremos internos de las cartelas, por lo que, el claro de cortante era igual a la longitud de las cartelas. Se estudiaron ángulos de acartelamiento entre $4.76^{\circ}$ y $16.70^{\circ}$, y se analizaron dos geometrías distintas para cada ángulo: 1) cuando el peralte menor está en los apoyos y, 2) cuando el peralte mayor es el de los apoyos. Cada serie contaba con uno o dos elementos prismáticos de referencia. En los experimentos de Debaiky y El-Niema (1982), la sección variable de las trabes no estaba en compresión, sino en tensión. Debaiky y El-Niema (1982) estudiaron el mecanismo resistente a cortante de sus trabes según el enfoque del ACI para elementos de sección constante y propusieron una ecuación donde de manera poco convincente, la cuantificación de la resistencia del acero de refuerzo transversal es igual que para trabes prismáticas (el agrietamiento diagonal se supuso $\theta=45^{\circ}$ ) y la contribución de refuerzo longitudinal inclinado lo atribuyen a la acción de dovela.

El-Niema (1988) reporta el ensaye de 10 especímenes de sección T simplemente apoyados con ángulos de $4.76^{\circ}$ y $9.46^{\circ}$ que básicamente su propósito principal era comprobar las recomendaciones hechas en Debaiky y El-Niema (1982). El-Niema (1988) no observó ningún cambio sustancial en el comportamiento de las trabes acarteladas con sección T respecto a las de sección rectangular, y concluyó que la resistencia a cortante de las trabes acarteladas con sección $\mathrm{T}$ podía calcularse con la fórmula propuesta por Debaiky y El-Niema, aunque no aclara si tomó o no en cuenta al patín de la sección T.

Stefanou (1983) estableció que la finalidad del acartelamiento es proveerse de una componente vertical del par interno de flexión que resista cortante, lo que provoca que una reducción del peralte no necesariamente reduzca la resistencia. Los desarrollos analíticos que presenta son importantes; sin embargo, la limitante de su estudio es que los experimentos que reporta son de 10 especímenes de sección rectangular simplemente apoyados de muy pequeño tamaño (claro efectivo de $1.22 \mathrm{~m}$, ancho de $10 \mathrm{~cm} \mathrm{y}$ peralte máximo de $15 \mathrm{~cm}$, reforzados con alambres). No obstante, quizás lo más importante de sus resultados experimentales es que de su análisis se observa un aumento en la resistencia a cortante del tramo acartelado cuando éste trabaja en compresión. 
Por otra parte, existe un estudio disponible para una condición de apoyo continuo (MacLeod y Houmsi 1994), pero tiene la limitante de haberse realizado también ante carga puntual estática monótona creciente y sólo para cinco cartelas sin refuerzo por cortante. Recientemente se ha publicado otro estudio que incluye trabes acarteladas continuas sin refuerzo por cortante (Pérez-Caldentey et al. 2012), pero está más orientado a evaluar el impacto del tipo de carga monótonamente creciente aplicada (puntual, uniformemente distribuida y triangularmente distribuida) en trabes (prismáticas y acarteladas), encontrando que la resistencia a corte sí depende fuertemente tanto del tipo de carga como de la condición de apoyo. Pérez-Caldentey et al. (2012) encontraron que la resistencia a cortante aumenta de manera importante cuando la cargas se aplican distribuidamente (carga uniforme y triangular) con respecto a las cargas aplicadas puntualmente. También confirman que la resistencia a cortante en condiciones de continuidad son mayores que en vigas simplemente apoyadas, como consecuencia que las deformaciones en la sección crítica por cortante es distinta en uno y otro caso.

Los únicos ensayes realizados hasta la fecha ante carga cíclica han sido en trabes acarteladas con condición de apoyo simple, con y sin refuerzo por cortante (Grande 2005 y 2009, Archundia et al. 2007, Tena-Colunga et al. 2007, Archundia-Aranda y Tena-Colunga 2008, Archundia-Aranda et al. 2013, Archundia 2013). Por todo lo anterior, no es sorprendente que en los principales libros especializados sobre el comportamiento y diseño de elementos de concreto reforzado no se trate el tema de trabes acarteladas, o esto se haga de una manera limitada (Park y Paulay 1997, Nielsen 1999, Nilson 1999, MacGregor y Wight 2005). Aunque en los libros de referencia se considera la participación del refuerzo longitudinal en el mecanismo resistente a cortante, lo cual es correcto, lo hacen bajo la hipótesis que las trabes acarteladas desarrollan patrones de agrietamiento por cortante similares a los de trabes prismáticas, lo cual no es preciso con base en la evidencia experimental.

Como consecuencia de la escasa información disponible sobre el comportamiento de trabes acarteladas de concreto reforzado, los reglamentos vigentes de diseño de estructuras de concreto más utilizados en México, como son las Normas Técnicas Complementarias para el Diseño de Estructuras de Concreto (NTCC-04 2004), y el reglamento ACI-318 (ACI-318-14 2014), no proporcionan recomendaciones específicas para diseño de elementos de concreto reforzado de sección variable, ni a cortante ni a flexión.

De esta manera, se puede afirmar que el diseño de trabes acarteladas en puentes y edificios en México (y en la mayor parte del mundo) ha dependido fundamentalmente de la experiencia y juicio del proyectista estructural. Salvo en contadas ocasiones, donde el diseño por cortante se ha basado directamente en planteamientos que toman en cuenta el mecanismo resistente a cortante por medio de modelos de puntales y tensores (Marti 1985, Dilger y Langohr 1997), en muchas ocasiones los diseñadores se han visto forzados a extrapolar su conocimiento sobre el comportamiento cíclico de elementos de geometría simple (prismáticos) a elementos de geometría más compleja (sección variable) ¿Será esto suficiente para garantizar el buen desempeño sísmico de estas construcciones y así salvaguardar la vida de quienes las habitan y/o circulan por ellas?

Existe evidencia fotográfica muy contundente que las trabes acarteladas son utilizadas muy frecuentemente en México en puentes y edificios, muchos de ellos ubicados en zonas sísmicas y particularmente en la ciudad de México y su zona conurbada (Tena-Colunga 1994, Archundia 2004 y 2013, Archundia et al. 2005, 2006 y 2007, Archundia-Aranda y Tena-Colunga 2008, Archundia-Aranda et al. 2013, Grande 2005 y 2009, Tena-Colunga et al. 2007 y 2008, López 2011, Urbina 2013), y que es escasa la investigación experimental básica que se requiere para entender, en primera instancia, su comportamiento ante carga cíclica reversible, con lo cual no se puede garantizar que su desempeño sismorresistente sea satisfactorio. Desafortunadamente, esto ya sucedió en la ciudad de México durante el 
sismo del 19 de septiembre de 1985, con las fallas por cortante que se apreciaron, entre otros, en el edificio de la Asociación Mexicana de Instituciones de Seguros (AMIS) ubicado en la calle de Londres, Colonia Juárez (Tena 1993, Tena-Colunga 1994) y en el edificio del antiguo cine Paseo, en el Paseo de la Reforma, ambos debidos a un detallado deficiente por cortante y, en el caso del edificio AMIS, a una pésima reparación previa por medio de encamisados parciales exclusivamente en las cartelas, por lo que la falla por cortante se produjo abruptamente al centro del claro de la zona prismática de las trabes acarteladas. Por ello, este grupo de investigación continúa desarrollando este esfuerzo e inició un programa experimental donde se están ensayando trabes acarteladas de concreto reforzado en condiciones de continuidad y sujetas a cargas cíclicas reversibles, que permitan estudiar su comportamiento a cortante inicialmente, para poder determinar cuáles son los parámetros más significativos que afectan su comportamiento. En las siguientes secciones se resumen sucintamente al programa experimental en curso y los resultados obtenidos del mismo en cuanto al comportamiento cíclico. En el artículo compañero (Tena et al. 2017) se discute con detalle el mecanismo resistente a cortante (evaluado experimentalmente) y la validez de ecuaciones que se han propuesto para su diseño, derivadas tanto del método del equilibrio en la sección crítica, como del método de puntales y tensores.

\section{DISEÑO POR CORTANTE DE LOS ESPECÍMENES DE PRUEBA}

Dado que al momento de iniciar este proyecto experimental no se contaba con los modelos de armadura desarrollados por Archundia (2013), todos los especímenes de prueba se diseñaron para fallar a cortante en condiciones de carga última según las recomendaciones propuestas en Tena-Colunga et al. (2008), pero con una modificación para tomar en cuenta la contribución del refuerzo inclinado con base en procesar resultados experimentales reportados por MacLeod y Houmsi (1994) para cartelas en doble voladizo sin refuerzo por cortante ensayadas estáticamente, donde se aprecian diferencias importantes en la contribución del refuerzo longitudinal inclinado a la resistencia a fuerza cortante con respecto a lo observado en trabes acarteladas simplemente apoyadas, como se ilustra en la figura 2.

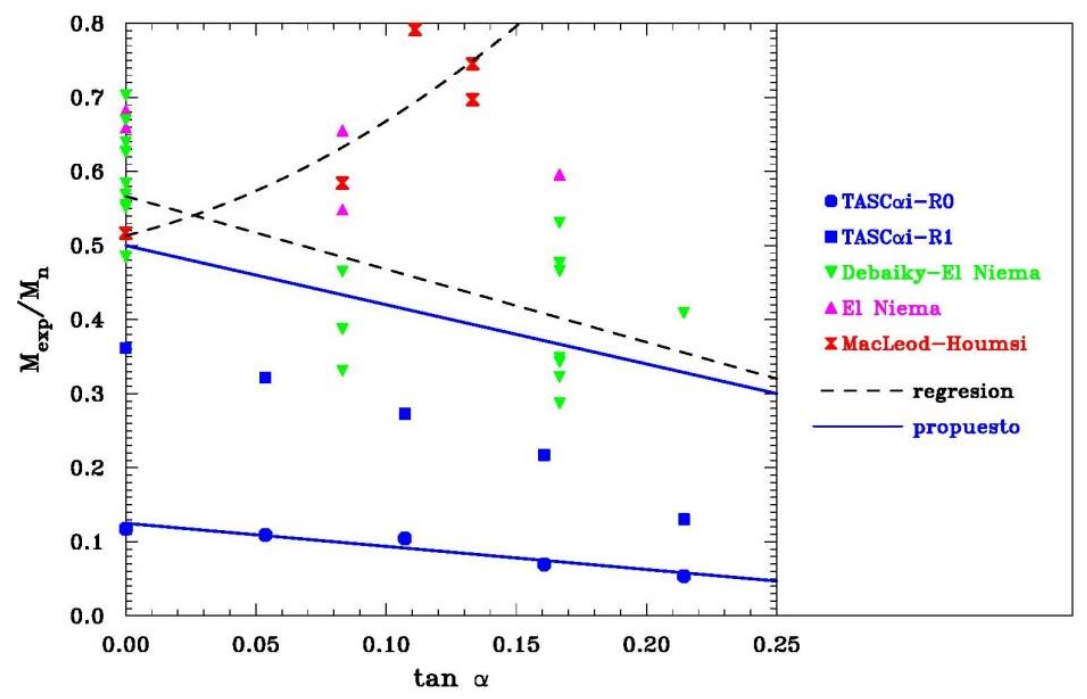

Figura 2. Contribución indirecta del refuerzo longitudinal inclinada a la resistencia a cortante de trabes acarteladas (ecuación de momento)

En la figura 2 se presenta una estimación de los momentos flexionantes desarrollados en la sección crítica que falló por cortante en distintos experimentos realizados en trabes acarteladas, que fueron normalizados con los momentos flexionantes teóricos si se desarrollara su capacidad a flexión en dicha sección, tal y como se describe con detalle en Tena-Colunga et al. (2008). En dicha figura, la inmensa 
mayoría de las trabes acarteladas ensayadas fueron en condición de apoyo simple, excepto las de MacLeod y Houmsi (1994), ensayadas en condiciones de continuidad. La mayoría de las trabes acarteladas contaban con un refuerzo mínimo por cortante, excepto las de MacLeod y Houmsi, y las identificadas como TASCai-R0, ensayadas por este grupo de investigación. Como se observa, con los escasos datos reportados en los experimentos de MacLeod y Houmsi, se estimó que se pueden desarrollar momentos flexionantes mayores en trabes acarteladas en condiciones de continuidad y sin contar con refuerzo por cortante, que los que se desarrollan en trabes acarteladas con refuerzo por cortante, pero en condición de apoyo simple, y esto impacta favorablemente la resistencia a cortante. Esto llamó un poco la atención, pues aunque se sabe desde hace tiempo conforme a la teoría (como se explica en el siguiente párrafo) que, en igualdad de circunstancias (geometría, cargas y refuerzo), la contribución del refuerzo inclinado es mayor en condiciones de continuidad (la cartela está sujeta a compresión) que de apoyo simple (la cartela trabaja a tensión), si sorprendió un poco que fuera mayor que en trabes simplemente apoyadas con refuerzo por cortante.

Se sabe conforme al desarrollo analítico presentado originalmente por Mörsch en 1908 (Archundia 2013), que la mayor diferencia en el mecanismo resistente a cortante de una trabe acartelada simplemente apoyada con respecto a una continua está dada por la contribución del refuerzo inclinado (por ejemplo, Mörsch 1952, Debaiky y El Niema 1982, El-Niema 1988, MacLeod y Houmsi 1994, Nilson 1999, Archundia 2004 y 2013, Tena-Colunga et al. 2008, Archundia et al. 2013, Urbina 2013, Archundia y Tena 2015) en función de su geometría y condiciones de carga, como se ilustra esquemáticamente con el apoyo de la figura 3. En la trabe acartelada continua se observa que el diagrama de momentos crece en el mismo sentido que la cartela (figura 3a), y en esta situación se suma la contribución del refuerzo inclinado $\left(V_{r i}\right)$ en la resistencia de la cartela a cortante $\left(V_{n}\right)$. En contraste, en una trabe acartelada simplemente apoyada, el diagrama de momentos crece en sentido contrario al que crece la cartela (figura 3b), por lo tanto, se resta la contribución del refuerzo inclinado en la resistencia de la cartela a cortante. En el diagrama de cortante, se observa también que en una trabe acartelada continua o en voladizo, el cortante máximo y el momento máximo coinciden, lo que no sucede en la viga simplemente apoyada.
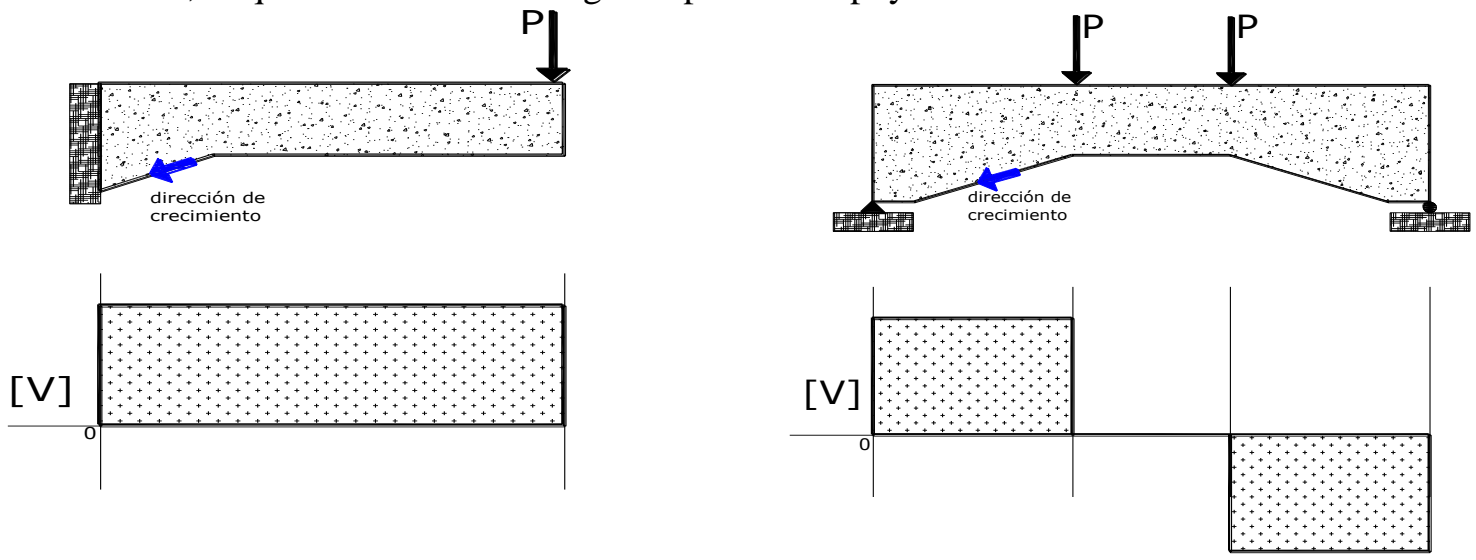

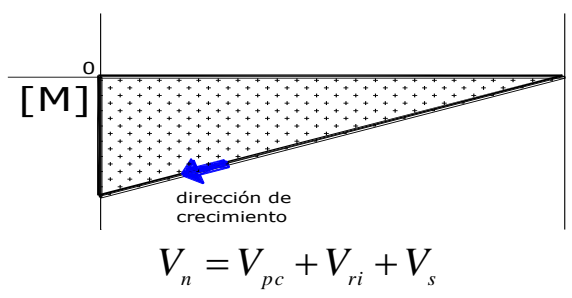

a) Condición de continuidad

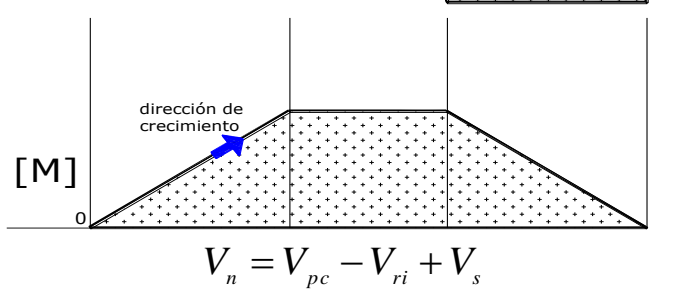

b) Simplemente apoyada

Figura 3. Resistencia teórica de trabes acarteladas para las condiciones de apoyo y carga ilustradas 
Lo anterior lo confirman las tendencias de las regresiones de los datos experimentales indicados en la figura 2 con líneas discontinuas, pues mientras que en los resultados de MacLeod y Houmsi (condición de continuidad, figura 3a) la relación $M_{\text {exp }} / M_{n}$ crece conforme aumenta el ángulo de acartelamiento (disminuye $\tan \alpha$ ), en el resto de los experimentos, en condiciones de apoyo simple (figura 3b), la relación $M_{\text {exp }} / M_{n}$ el momento decrece conforme aumenta el ángulo de acartelamiento.

Cabe señalar que en términos más generales, independientemente de las condiciones de apoyo, la contribución del refuerzo inclinado a la resistencia a cortante de una trabe acartelada se suma cuando el peralte de la cartela crece en el mismo sentido que el diagrama de momentos, y se resta cuando el peralte de la cartela y el diagrama de momentos crecen en direcciones opuestas. Por lo tanto, existen casos para trabes acarteladas en condiciones de apoyo simple donde se deba sumar la resistencia del refuerzo inclinado, por ejemplo, cuando bajo la condición de carga ilustrada en la figura 3b, tuviera su mayor peralte al centro del claro, y de trabes continuas donde se deba restar la resistencia del refuerzo inclinado, por ejemplo, cuando bajo la condición de carga ilustrada en la figura $3 \mathrm{a}$, tuviera su menor peralte en el extremo.

Por lo tanto, el diseño por cortante de las trabes acarteladas en condiciones de continuidad se hizo de la siguiente manera. La resistencia nominal a cortante de las trabes, $V_{n}$, se determinó como:

$$
\begin{aligned}
& V_{n}=V_{c}+V_{s} \\
& V_{c}=V_{p c}+V_{r i}
\end{aligned}
$$

donde $V_{c}$ es la contribución total del concreto, que toma en cuenta la potencial contribución del refuerzo inclinado y por efecto de dovela, $V_{p c}$ es la contribución nominal del concreto, $V_{r i}$ es la contribución del acero de refuerzo longitudinal inclinado, y $V_{s}$ es la contribución del acero de refuerzo transversal (estribos). La contribución nominal del concreto $V_{p c}$, conforme lo propone el reglamento ACI-318, está dada por:

$$
V_{p c}=\left(0.5 \sqrt{f_{c}^{\prime}}+176 \rho_{w} \frac{V_{n} d_{c r}}{M_{n}}\right) b d_{c r}
$$

donde $b$ es el ancho de la sección transversal, $f_{c}^{\prime}$ es la resistencia nominal a compresión del concreto, $\rho_{w}$ es la cuantía de refuerzo longitudinal, $V_{n}$ y $M_{n}$ son, respectivamente, el cortante nominal y momento nominal aplicados a la sección crítica, y $d_{c r}$ es el peralte crítico por cortante en las cartelas y se calcula de la siguiente forma (Archundia 2004, Tena-Colunga et al. 2008):

$$
d_{c r}=d_{\text {min }}[1+1.35 \tan \alpha] \leq\left[\left(\frac{h_{\max } h_{\min }-h_{\max }^{2}}{2 l_{h}}+h_{\max }\right)-r\right]
$$

donde $\alpha$ es el ángulo de acartelamiento, $d_{\min }$ es el peralte efectivo menor en las cartelas, $h_{\operatorname{máx}} \mathrm{y} h_{\min }$ es el peralte total máximo y mínimo en las cartelas, $l_{h}$ es la longitud de acartelamiento y $r$ es el recubrimiento del concreto para el refuerzo longitudinal.

Para calcular la contribución del acero de refuerzo transversal en la resistencia a la fuerza cortante, $V_{s}$, se utilizó la ecuación propuesta en Tena-Colunga et al. (2008): 


$$
V_{s}=\frac{A_{v} f_{y v} d_{c r}}{s \tan (45-\alpha)}
$$

donde $\alpha$ es el ángulo de acartelamiento y $A_{v}$ es la suma de las áreas de las ramas de los estribos separados una distancia, $s$. El peralte, $d_{c r}$, se calcula conforme a la ecuación (4), y $f_{y v}$ es el esfuerzo de fluencia del acero del refuerzo transversal.

La contribución del acero de refuerzo longitudinal inclinado, $\mathrm{V}_{r i}$, se calcula como:

$$
V_{r i}= \pm \frac{M_{c r}}{d_{c r}} \tan \alpha
$$

donde $\alpha$ es el ángulo de acartelamiento, el peralte $d_{c r}$ se obtiene de la ecuación 3, y $M_{c r}$ es el momento flexionante que se desarrolla en la sección crítica cuando se produce una falla por cortante, que para los ensayes realizados en trabes acarteladas con refuerzo transversal y en condiciones de apoyo simple, en Tena-Colunga et al. (2008) se propuso estimar como (figura 2):

$$
M_{c r}=0.5 M_{n}(1-1.6 \tan \alpha)
$$

donde $M_{n}$ es el momento flexionante nominal en la sección crítica, si la falla es por flexión y no por cortante.

Conforme a lo crudamente estimado de los datos experimentales de MacLeod y Houmsi, de la figura 2 se aprecia que aún sin refuerzo por cortante, la capacidad a flexión en la sección crítica es importante y aumenta conforme aumenta el ángulo de acartelamiento. Por lo tanto, a falta de mejor información, se propuso para trabes acarteladas en condiciones de continuidad estimar preliminarmente y de manera no conservadora la resistencia a cortante con la ecuación 8 por la siguiente razón. En la planeación de los experimentos es esencial revisar la capacidad del piso de reacción (para asegurar que permanezca elástico), así como determinar las características y capacidades mínimas de los gatos hidráulicos a emplear en el ensaye.

$$
M_{c r}=0.85 M_{n}(1+1.6 \tan \alpha) \leq M_{n}
$$

Finalmente, en los vértices se proveyó un refuerzo transversal para resistir la componente vertical que se produce por el cambio de dirección del acero longitudinal inferior, como se ilustra en la figura 4, donde la componente vertical $\left(F_{v}\right)$ que produce el cambio de dirección de acero longitudinal se estima como:

$$
F_{v}=A_{s} f_{y} \operatorname{sen} \alpha \leq T_{y} \operatorname{sen} \alpha
$$

donde $T_{y} \operatorname{sen} \alpha$ es la fuerza de tensión del acero longitudinal en la fluencia, As es el área de acero longitudinal y $\alpha$ es el ángulo de inclinación de la cartela con respecto a un eje horizontal. 

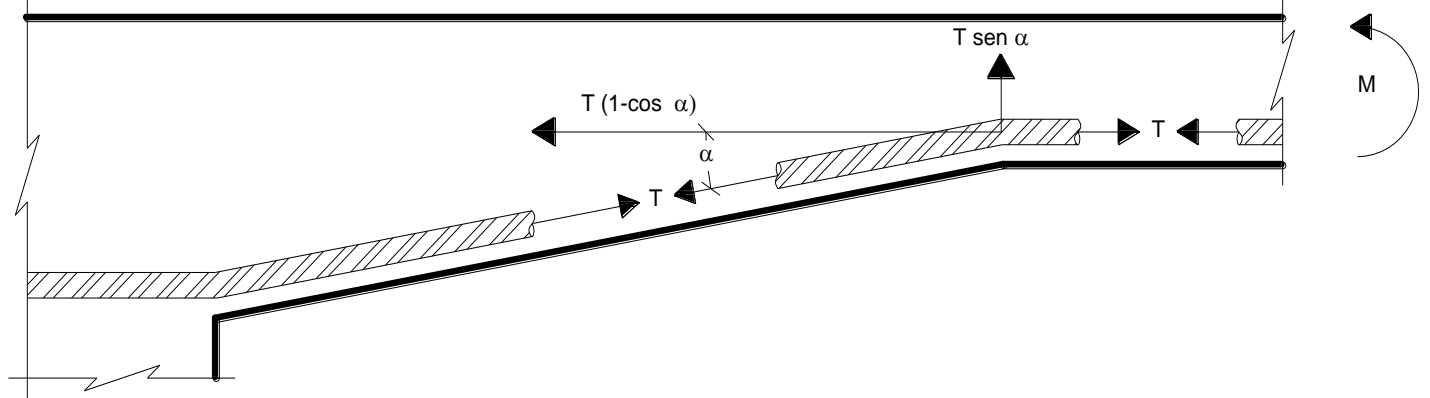

Figura 4. Fuerzas por el cambio de dirección de refuerzo horizontal

La separación del refuerzo transversal requerido en la zona donde se produce el cambio de dirección del acero longitudinal, $S_{c d}$ (en el vértice y el nudo, figura 5), se obtiene como:

$$
S_{c d}=\frac{A_{v} f_{y v} d_{\min }}{F_{v}}
$$

donde $d_{\min }$ es el peralte efectivo en la zona prismática de la viga, $A_{v}$ es la suma de las áreas de las ramas de los estribos, y $f_{y v}$ es el refuerzo de fluencia del acero del refuerzo transversal.

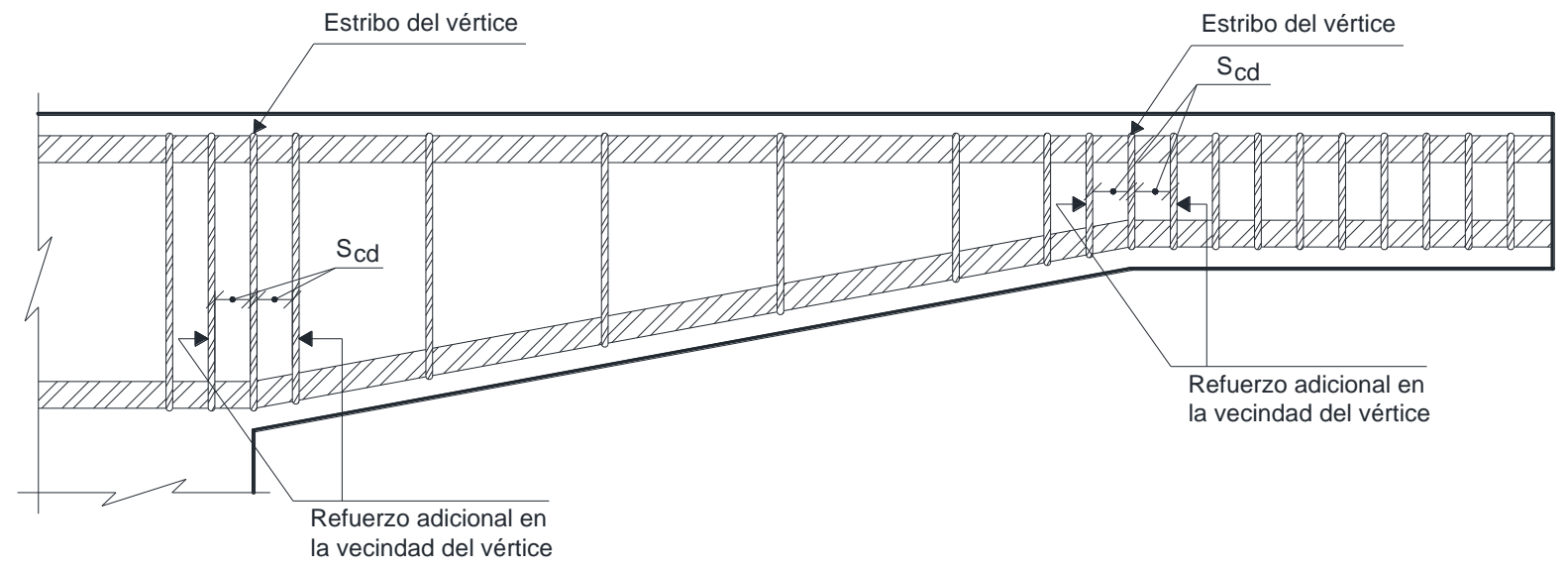

Figura 5. Refuerzo transversal en los vértices de la trabes

\section{Geometría de los especímenes de prueba}

El tamaño de las trabes acarteladas de concreto reforzado ensayadas en condiciones de continuidad se fijó con base en la capacidad de carga y maniobras del Laboratorio de Modelos Estructurales Intermedios de la UAM-Azcapotzalco, la cual se planeó y revisó como se reporta en López (2011).

Por las restricciones importantes que existe en la grúa del laboratorio (con capacidad de tres toneladas máximo), además de tomar en cuenta la geometría empleada en los ensayes realizados por MacLeod y Houmsi, en los especímenes de prueba se considera un nudo típico interior de un marco en el último nivel (figura 6), por lo que las secciones de trabes en estudio corren del nudo al centro del claro teórico. 


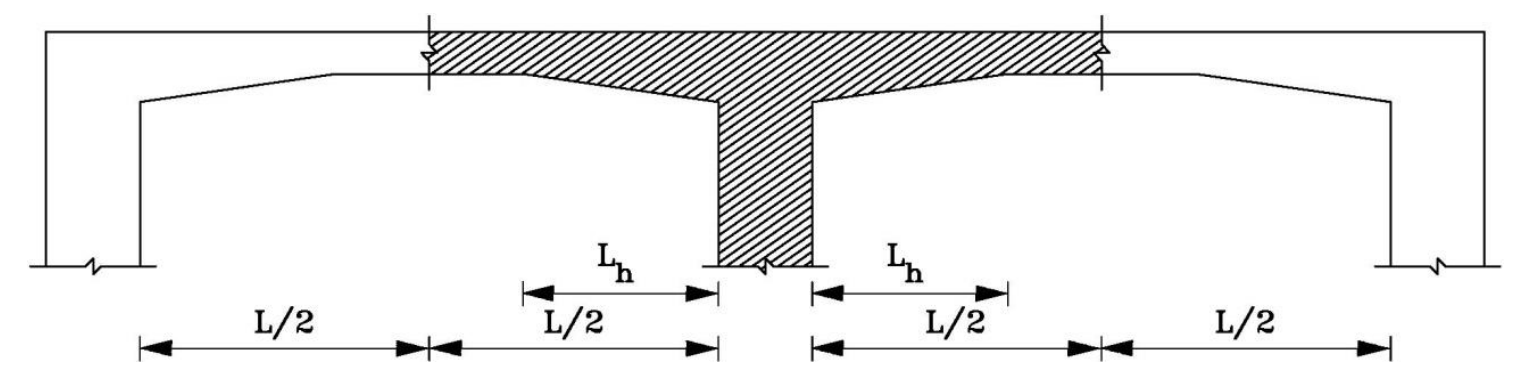

Figura 6. Espécimen de prueba (sombreado) representativo de un marco interior de último nivel

Por lo tanto, se propusieron especímenes de prueba cuya longitud total es de $4.3 \mathrm{~m}$ (figura 7), con un claro equivalente a paños de columna (luz) de la crujía del marco $(L)$ de $3.7 \mathrm{~m}$. El ancho de las trabes fue $b=25 \mathrm{~cm}$ y es constante para todos los especímenes. El peralte total máximo, que corresponde al nudo viga-columna es siempre $h_{\max }=45 \mathrm{~cm}$, y el peralte mínimo $h_{\min }$, que corresponde a la sección prismática de la trabe, varía entre $45 \mathrm{~cm}$ (trabe prismática, $\left.\alpha=0^{0}\right)$ y $23 \mathrm{~cm}\left(\alpha=9.98^{\circ}\right)$.

La columna de concreto reforzado del marco tiene una sección transversal rectangular de $60 \mathrm{~cm} \mathrm{x}$ $45 \mathrm{~cm}$. La longitud de las columnas varía de acuerdo con el grado de acartelamiento, y esto se hizo en función de la carrera de los gatos hidráulicos a emplear en los ensayes, con la finalidad de tener suficiente capacidad para empujar y jalar a los especímenes. La columna está anclada a un dado o muerto de concreto, que a su vez estará sujeto al piso de reacción.

En la figura 7 se muestra un croquis esquemático de las dimensiones de las trabes acarteladas en doble voladizo ensayadas.

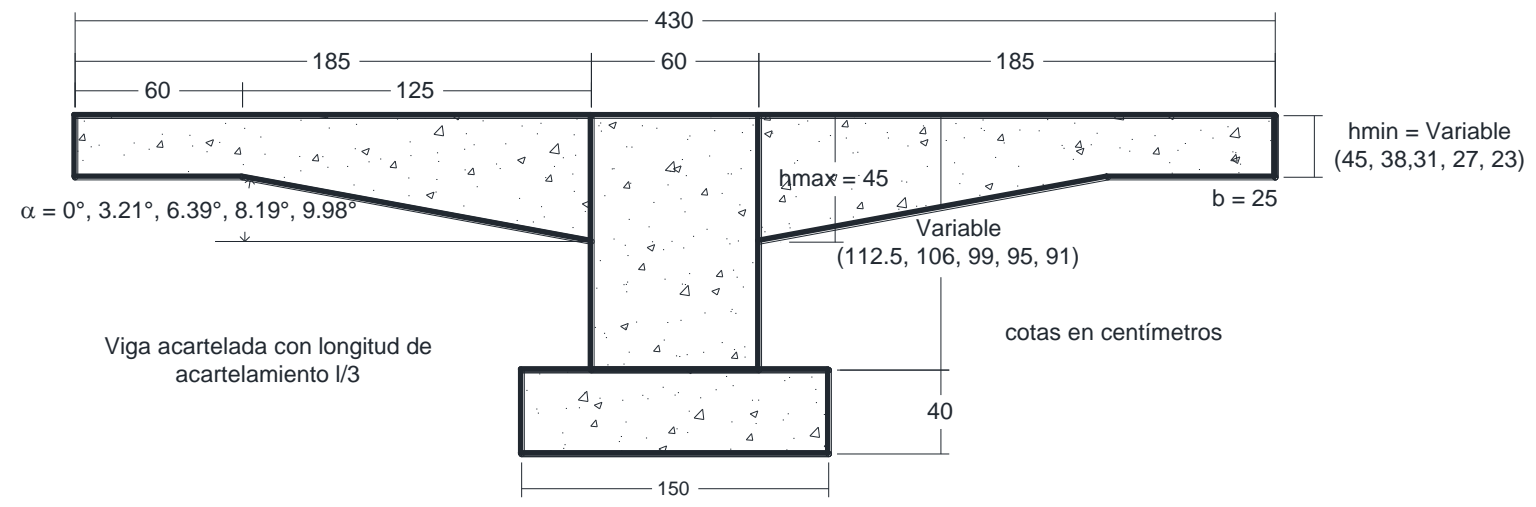

Figura 7. Dimensiones generales de los especímenes de prueba (acotaciones: $\mathrm{cm}$ )

El muerto se sujetó al piso de reacción mediante 12 anclas de una pulgada de diámetro, 12 tubos conduit galvanizado de pared delgada de 1/1/4" ahogados en el dado de concreto, una placa base de acero de $1 / 2$ " de espesor con medidas de $180 \mathrm{~cm}$ x $80 \mathrm{~cm}$, y dos placas de acero de $1 / 2$ " de espesor de $10 \mathrm{~cm}$ x 150 $\mathrm{cm}$. Este anclaje fue diseñado para que los especímenes, al ser sometidos a las cargas cíclicas de empuje y jale, no presentaran ni volteo ni aplastamiento.

Para la identificación de cada una de la trabes acarteladas, se siguió la nomenclatura de ensayes experimentales previos (por ejemplo, Archundia 2004, Archundia et al. 2005, Grande 2005, Tena-Colunga et al. 2008, Grande 2009, Archundia-Aranda et al. 2013, Archundia 2013). La nomenclatura utilizada 
permite identificar a los elementos según su refuerzo transversal, refuerzo longitudinal, ángulo de acartelamiento y tipo de carga aplicada. La nomenclatura, que se ilustra en la figura 8, está formada por once literales, de los cuales los que están en negritas son constantes, mientras que los que están en negritas y cursivas son variables.

El significado de la nomenclatura es el siguiente. Los primeros caracteres de la primer literal (TA), indican que se trata de trabes acarteladas. La tercera literal indica el tipo de falla que se espera en el elemento, $\boldsymbol{S}$ significa una falla por cortante (del inglés shear). La cuarta literal identifica el arreglo del refuerzo longitudinal, la opción $\boldsymbol{C}$ se refiere al arreglo continuo (único utilizado en este trabajo). La quinta literal, $(\boldsymbol{V})$, indica que esta en condición de continuidad (doble voladizo). La sexta (3), indica la longitud de acartelamiento utilizada: 3 , ya que para este trabajo de investigación se utilizó L/3. La séptima y octava literales $(\boldsymbol{\alpha} \boldsymbol{i})$ identifican a los diferentes ángulos de acartelamiento $\alpha$ utilizados en este trabajo experimental. El contador (i) adopta los valores siguientes: $\boldsymbol{0}$ para un ángulo $\alpha=0^{\circ}$ (trabe prismática), $\boldsymbol{I}$ para $\alpha=3.21^{\circ}, 2$ para $\alpha=6.39^{\circ}, 3$ para $\alpha=8.19^{\circ}$ y 4 para $\alpha=9.98^{\circ}$. La novena y décima literales $(\mathbf{R j})$ identifican el tipo de refuerzo transversal para resistir cortante, $\mathbf{R}$. El contador $(j)$ adopta los valores siguientes: $\boldsymbol{O}$ para los elementos sin refuerzo transversal y $\mathbf{1}$ para los elementos que contienen un refuerzo transversal mínimo por cortante. La undécima literal identifica el tipo de carga a aplicar, $\boldsymbol{c}$ significa carga cíclica.

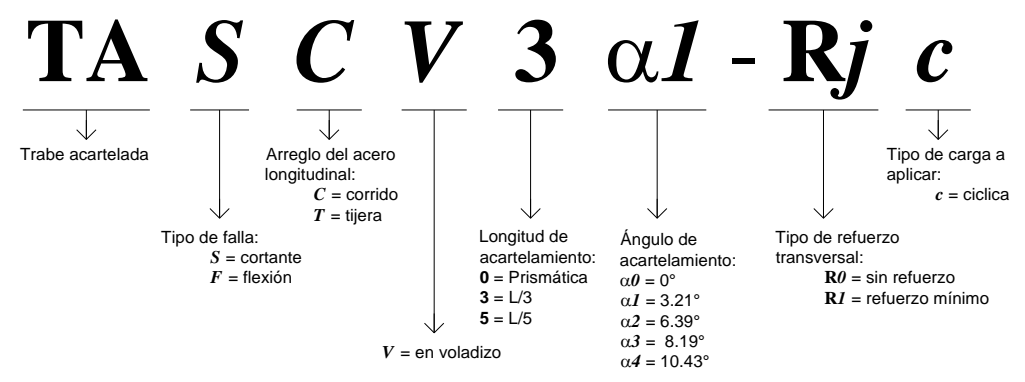

Figura 8. Nomenclatura empleada para la identificación de los especímenes

El diseño del refuerzo longitudinal y transversal de las trabes acarteladas se hizo para garantizar que se desarrollara una falla por cortante en la zona de la cartela (y no por flexión). Se consideró una resistencia nominal del concreto a la compresión $f_{c}^{\prime}=250 \mathrm{~kg} / \mathrm{cm}^{2}$ y un esfuerzo nominal de fluencia del acero de refuerzo $f_{y}=4,200 \mathrm{~kg} / \mathrm{cm}^{2}$. Sin embargo, también se tomó en cuenta que la resistencia del concreto a la hora del ensaye puede ser superior a la nominal, y con base en la experiencia de ensayes previos, se estimaron resistencias también para una resistencia $f_{c}^{\prime}=300 \mathrm{~kg} / \mathrm{cm}^{2}$. Asimismo, se emplearon también los esfuerzos de fluencia "reales" del acero de refuerzo obtenidos en ensayes previos (por ejemplo, Archundia 2004, Grande 2005, Tena-Colunga et al. 2008, Grande 2009, Archundia-Aranda et al. 2013) que en promedio fueron $f_{y}=4,348 \mathrm{~kg} / \mathrm{cm}^{2}$ para el acero longitudinal y $f_{y v}=4,592 \mathrm{~kg} / \mathrm{cm}^{2}$ para el acero transversal.

Dada la geometría de los especímenes de ensaye (figura 7), para garantizar la falla por cortante en la viga, no sólo es importante vigilar que esto sucediera en la sección crítica, sino que la falla no ocurriera en el vértice, ni en el nudo. Por lo que se propusieron y evaluaron tres factores de seguridad globales, como se describe en Tena et al. (2014).

El diseño de la columna, del nudo y del muerto se realizó conforme a las NTCC-04 (2004), para garantizar que éstos fueran los elementos más fuertes del ensaye, utilizando los criterios que se describen y reportan con mayor detalle en López (2011) y Urbina (2013). 
Conforme a todo lo expuesto anteriormente, se definieron los armados de los especímenes que se reportan con detalle en Urbina (2013), y que se sintetizan en la tabla 1 sólo para las trabes acarteladas y que se muestran en las figuras 9 a 13. El refuerzo típico de las secciones transversales máximas y mínimas de las trabes acarteladas se muestra en la figura 14 y de la columna en la figura 15.

En la tabla 2 se reportan las resistencias nominales a cortante obtenidas para cada cartela sujetas a momento positivo y negativo, cuando se consideran resistencias superiores a las especificadas en el diseño: $f_{c}^{\prime}=300 \mathrm{~kg} / \mathrm{cm}^{2}$ para el concreto, $f_{y}=4,348 \mathrm{~kg} / \mathrm{cm}^{2}$ para el acero de refuerzo longitudinal y $f_{y v}=$ $4,592 \mathrm{~kg} / \mathrm{cm}^{2}$ para el acero de refuerzo transversal, considerando las ecuaciones 1 a 6 , y la ecuación 8 para estimar la contribución del refuerzo longitudinal inclinado.

Tabla 1. Resumen del armado de las trabes acarteladas en estudio

\begin{tabular}{|c|c|c|c|c|c|c|c|}
\hline \multirow{2}{*}{ Espécimen } & \multirow{2}{*}{$\alpha$} & \multicolumn{2}{|c|}{ Refuerzo Longitudinal } & \multicolumn{4}{|c|}{ Refuerzo por Cortante } \\
\hline & & Superior & Inferior & Extremos & Cartelas & Vértice & Paño-Viga-Col. \\
\hline TASCV3a0-R1c & $0^{\circ}$ & $3 \# 12$ & $2 \# 12$ & $\begin{array}{l}\text { 9E\#3@6 cm } \\
5 \mathrm{E} \# 3 @ 10 \mathrm{~cm}\end{array}$ & 4E\#3@ $25 \mathrm{~cm}$ & - & - \\
\hline TASCV3 $\alpha 1-R 1 c$ & $3.21^{\circ}$ & $3 \# 12$ & $2 \# 12$ & 9E\#3@6 cm & 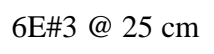 & - & - \\
\hline TASCV3 $\alpha 2-R 1 c$ & $6.39^{\circ}$ & $3 \# 12$ & $2 \# 12$ & 9E\#3@6 cm & $6 \mathrm{E \# 3} @ 25 \mathrm{~cm}$ & 2E\#3@12.5cm & 2E\#3@12.5cm \\
\hline TASCV3a3-R1c & $8.19^{\circ}$ & $3 \# 12$ & $2 \# 12$ & 9E\#3@6 cm & 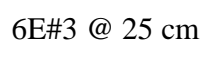 & 2E\#3@10cm & 2E\#3@10cm \\
\hline TASCV3a4-R1c & $9.98^{\circ}$ & $3 \# 12$ & $2 \# 12$ & 9E\#3@6 cm & 6E\#3@ @25 cm & 4\#3@6cm & 4\#3@6cm \\
\hline
\end{tabular}

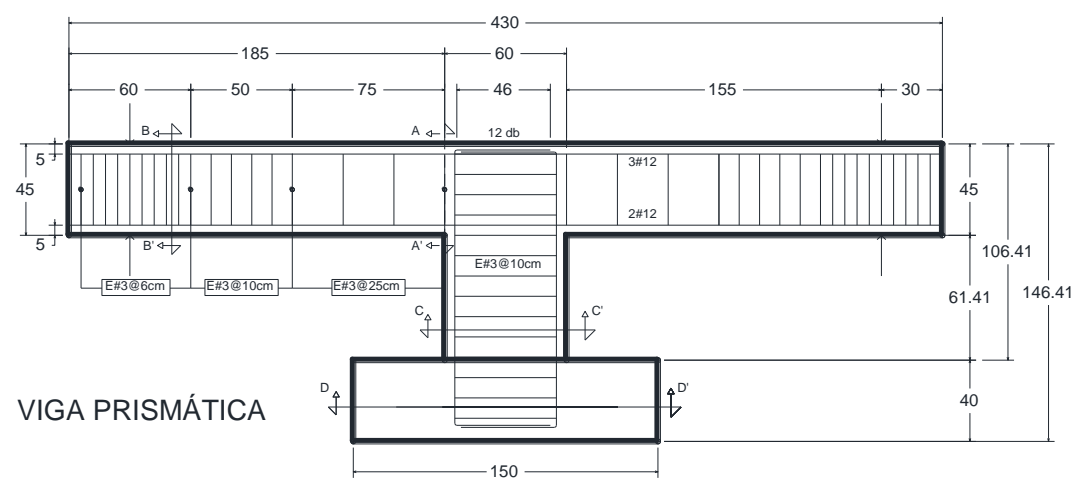

Figura 9. Armado del espécimen TASCV3 $\alpha 0-\mathrm{R} 1 \mathrm{c}$ (acotaciones en $\mathrm{cm}$ )

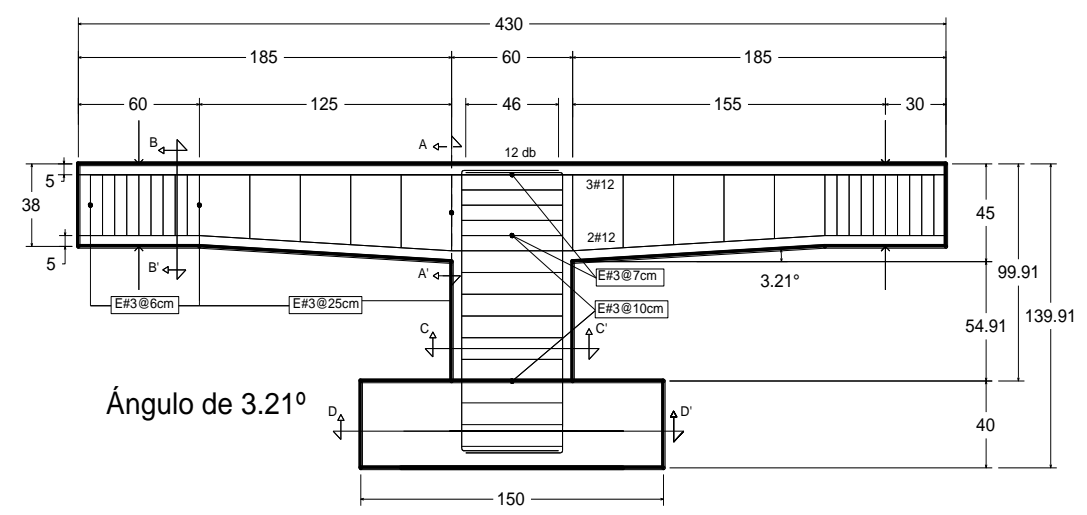

Figura 10. Armado del espécimen TASCV3a1-R1c (acotaciones en cm) 


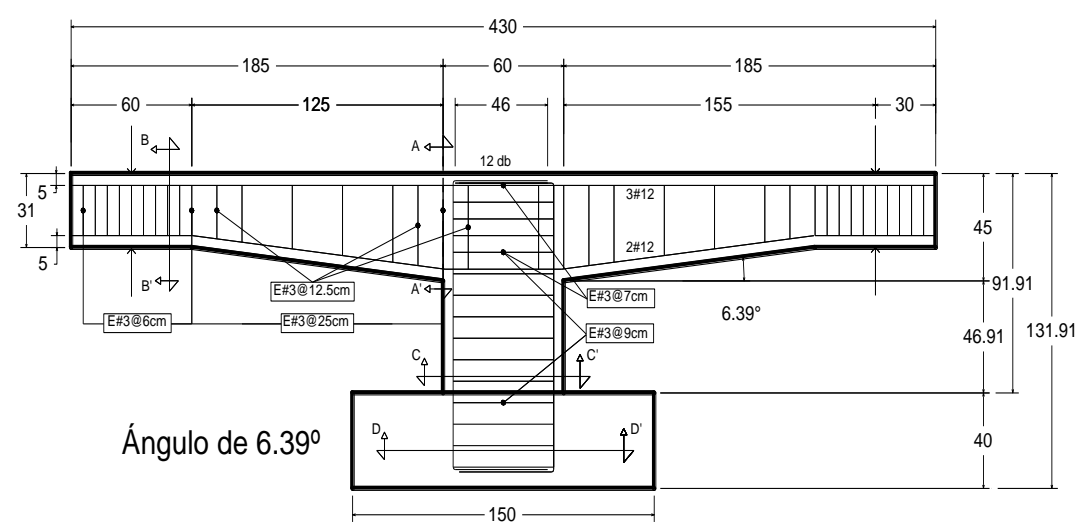

Figura 11. Armado del espécimen TASCV3 $\alpha 2-\mathrm{R} 1 \mathrm{c}$ (acotaciones en $\mathrm{cm}$ )

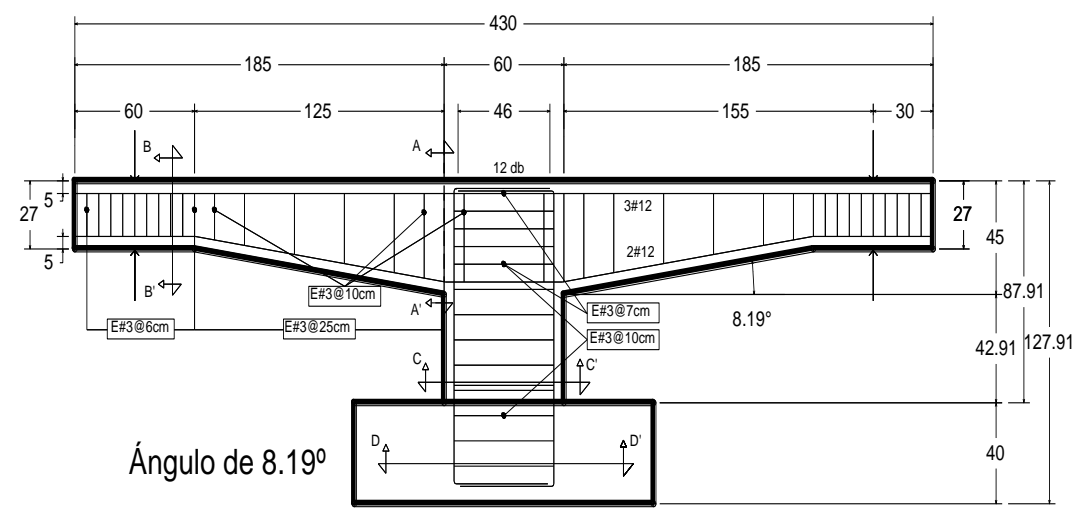

Figura 12. Armado del espécimen TASCV3a3-R1c (acotaciones en $\mathrm{cm}$ )

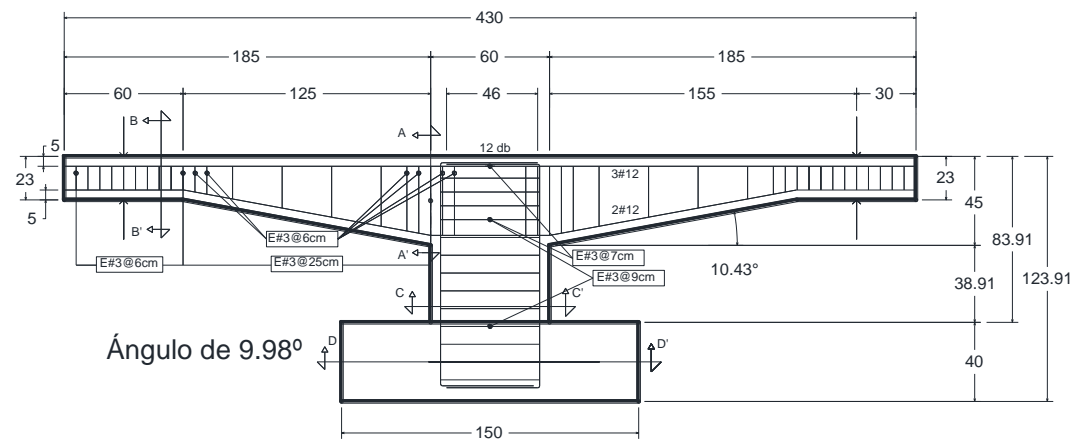

Figura 13. Armado del espécimen TASCV3a4-R1c (acotaciones en $\mathrm{cm}$ )

Se pueden hacer varias observaciones importantes de los resultados mostrados en la tabla 2. El primero es que a diferencia de lo que sucede en trabes prismáticas, en teoría, en las trabes acarteladas si existe una diferencia apreciable en cuanto a su capacidad a resistir cortante $\left(V_{n}\right)$ ante momento positivo y negativo cuando el acero de refuerzo del lecho superior e inferior es distinta (figura 14), como consecuencia de la contribución del refuerzo longitudinal inclinado $\left(V_{r i}\right)$. En este caso, dado que el refuerzo longitudinal del lecho superior es mayor (figura 14), la capacidad a momento negativo es mayor $\mathrm{y}$, por ende, el cortante que resiste a momento negativo en condición de continuidad es mayor. También se aprecia que, en teoría, la contribución al cortante del refuerzo longitudinal inclinado $\left(V_{r i}\right)$ puede llegar a 
ser casi tan o más importante que la contribución nominal del concreto $\left(V_{p c}\right)$ para ángulos de seis grados (espécimen TASCV3 $\alpha 2-\mathrm{R} 1 \mathrm{c}$ ) o mayores. La tercera, y no menos importante, es que cuando la geometría de la cartela favorece que el refuerzo longitudinal inclinado sume a la resistencia al corte, como es el caso en estudio, en teoría, la resistencia a cortante es superior que el de una trabe prismática que tenga el mismo peralte máximo. Esta es una observación muy importante, que será corroborada posteriormente con los resultados de los experimentos que aquí se presentan.

Tabla 2. Resistencias nominales a cortante (toneladas) considerando fuentes de sobrerresistencia

\begin{tabular}{|c|c|c|c|c|c|c|c|c|}
\hline \multirow[t]{2}{*}{ Espécimen } & \multicolumn{4}{|c|}{ Momento Positivo } & \multicolumn{4}{|c|}{ Momento Negativo } \\
\hline & $V_{n}$ & $V_{p c}$ & $V_{s}$ & $V_{r i}$ & $V_{n}$ & $V_{p c}$ & $V_{s}$ & $V_{r i}$ \\
\hline TASCV3 $\alpha 0-R 1 c$ & 20.00 & 9.57 & 10.43 & - & 20.02 & 9.59 & 10.43 & - \\
\hline TASCV3 $\alpha 1-R 1 c$ & 22.89 & 8.74 & 10.36 & 3.79 & 24.74 & 8.85 & 10.36 & 5.53 \\
\hline TASCV3 $\alpha 2-R 1 c$ & 24.04 & 7.61 & 9.78 & 6.65 & 27.23 & 7.81 & 9.78 & 9.65 \\
\hline TASCV3 $\alpha 3-R 1 c$ & 23.83 & 6.84 & 9.16 & 7.84 & 27.53 & 7.07 & 9.16 & 11.30 \\
\hline TASCV3 $\alpha 4-R 1 c$ & 23.43 & 6.03 & 8.51 & 8.89 & 27.51 & 6.31 & 8.51 & 12.70 \\
\hline
\end{tabular}

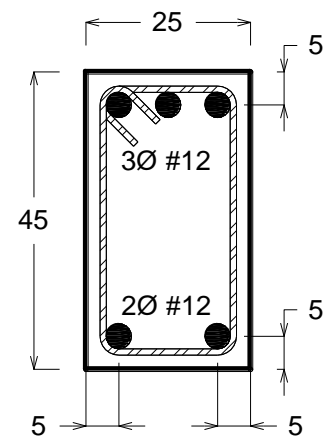

Figura 14. Armados en las secciones transversales máximas y mínimas de las cartelas

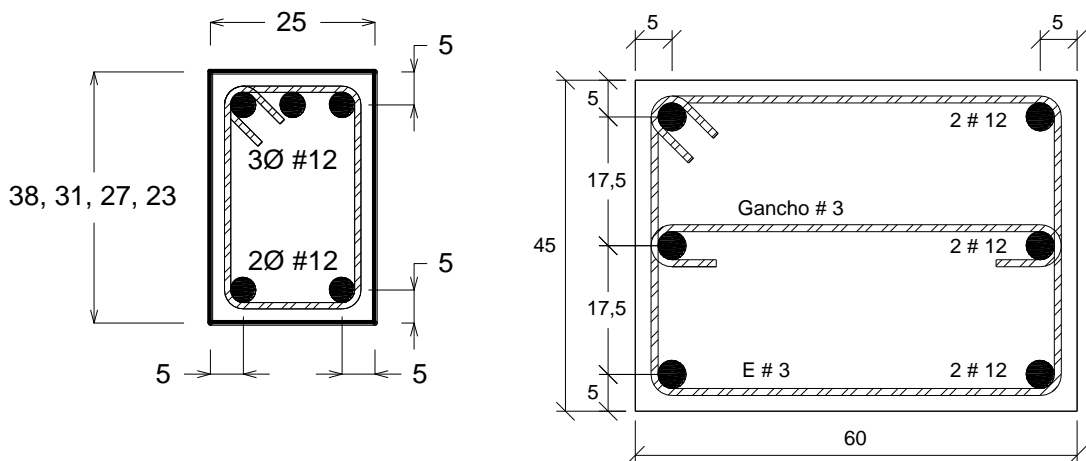

Figura 15. Armado de la sección transversal típica de las columnas

\section{ENSAYES EXPERIMENTALES}

Los especímenes de prueba se construyeron en el Laboratorio de Modelos Estructurales Intermedios de la UAM-A, con el apoyo de numerosos alumnos de la Licenciatura en Ingeniería Civil. Los detalles sobre el proceso constructivo se reportan en Urbina (2013) y Aranda (2015).

\section{Instrumentación}

Todas las trabes en estudio se instrumentaron internamente por medio de deformímetros o galgas extensométricas (strain gages), los cuales oscilaron entre 54 y 58, conforme se detalla en la tabla 3 . En la figura 16 se ejemplifica la instrumentación interna típica de los especímenes. 
Tabla 3. Instrumentación interna de cada uno de los especímenes en estudio

\begin{tabular}{|c|c|c|c|c|c|c|}
\hline \multirow[b]{2}{*}{ Espécimen } & \multirow[b]{2}{*}{$\begin{array}{c}\text { Total de } \\
\text { Deformímetros }\end{array}$} & \multirow{2}{*}{$\begin{array}{c}\text { Cartela } \\
\text { Izquierda }\end{array}$} & \multirow[b]{2}{*}{ Cartela Derecha } & \multicolumn{3}{|c|}{ Columna } \\
\hline & & & & $\begin{array}{c}\text { Parte } \\
\text { superior }\end{array}$ & $\begin{array}{c}\text { Parte } \\
\text { Inferior }\end{array}$ & $\begin{array}{c}\text { Parte } \\
\text { Central }\end{array}$ \\
\hline TASCV0a0-R1c & 58 & 23 & 23 & 5 & 3 & 4 \\
\hline TASCV3a1-R1c & 54 & 21 & 21 & 5 & 3 & 4 \\
\hline TASCV3a2-R1c & 54 & 21 & 21 & 5 & 3 & 4 \\
\hline TASCV3a3-R1c & 54 & 21 & 21 & 5 & 3 & 4 \\
\hline TASCV3a4-R1c & 58 & 23 & 23 & 5 & 3 & 4 \\
\hline
\end{tabular}

La instrumentación externa de los especímenes consistió en transductores de desplazamiento (TI-i y TD-i), micrómetros de carátula (TRI y TRD), deformímetros de concreto (SGCi) y celdas de carga. Los transductores de desplazamiento se colocaron en la parte inferior y a lo largo de cada cartela. El primero se colocó al inicio de la trabe, el segundo en el vértice de cambio de sección de prismática a acartelada, y el tercero a una distancia de $45 \mathrm{~cm}$ del paño exterior del nudo viga-columna hacia la trabe, como se ilustra en la figura 17. Los resultados obtenidos para todos los puntos de medición se reportan en Urbina (2013).

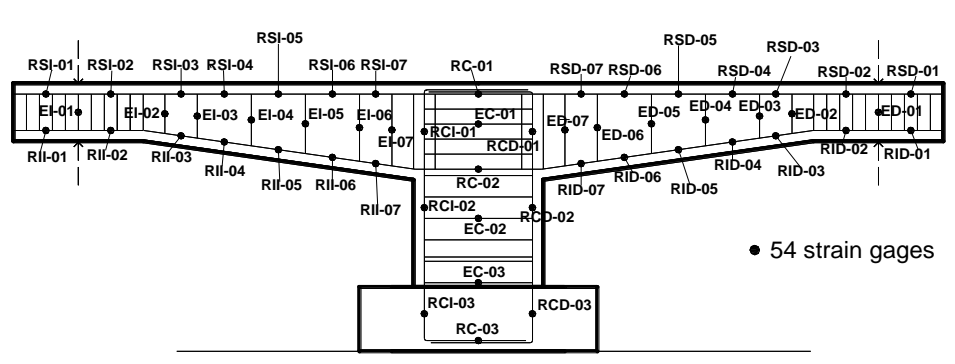

a) Ubicación de deformímetros

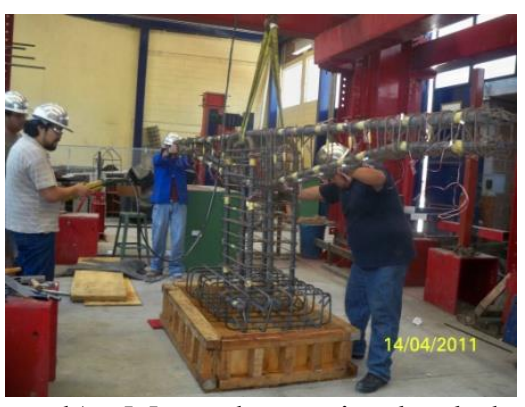

b) Montado previo al colado

Figura 16. Instrumentación interna del espécimen TASCV3 $\alpha 3-\mathrm{R} 1 \mathrm{c}$

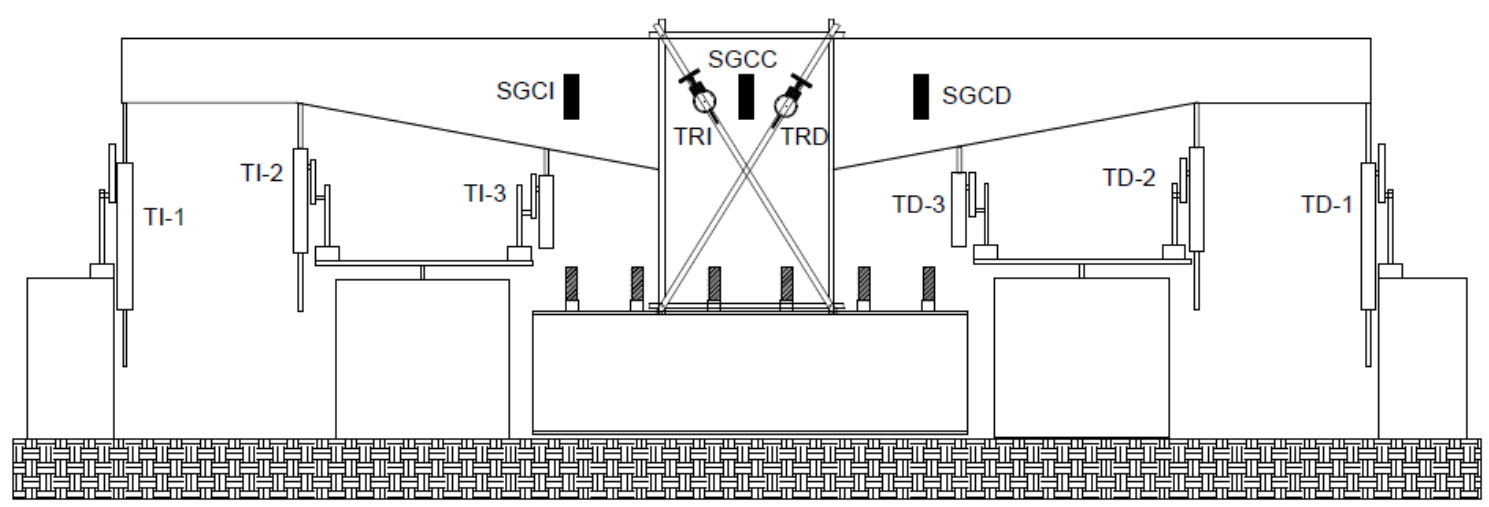

Figura 17. Esquema de instrumentación externa de los especímenes

\section{Aplicación de cargas cíclicas}

El dispositivo de ensaye fue diseñado para aplicar cargas reversibles (figura 18), y consistió en dos placas dobles de acero en la parte superior y en la parte inferior en cada extremo del espécimen, unidas mediante cuatro barras roscadas de $1 \frac{1}{2} 2$ pulgada de diámetro por extremo y tensadas mediante tuercas. A las placas inferiores se les soldó una placa de acero de 11/2 pulgada de espesor para darle estabilidad y 
rigidez a la misma. A estas placas se les soldó la conexión para la aplicación de la carga cíclica. La conexión se compuso de dos tramos de tubo de acero con diámetro exterior de cuatro pulgadas y diámetro interior de tres pulgadas con una longitud de $17 \mathrm{~cm}$ cada uno, un muñón de acero A36, el cual tiene una perforación de tres pulgadas en su parte central. En su parte inferior cuenta con un apéndice de 13/4 de pulgada de diámetro y cinco centímetros de largo, y un perno de tres pulgadas de diámetro con una longitud de $85 \mathrm{~cm}$, que atraviesa los tubos y la parte central del muñón. El muñón se conectó en su parte inferior a una celda de carga, y a su vez la celda de carga se conectó a un actuador de doble acción. El gato hidráulico se sujetó a dos placas soldadas de $3 / 4$ de pulgada de espesor atiesadas por medio de cartabones de $1 / 2$ pulgada de espesor (figura 18 ).

Las cargas se aplicaron por medio de gatos hidráulicos de doble acción (figura 18) con capacidad de 95 toneladas; éstos fueron accionados con un equipo de bombeo, provisto de válvulas de paso para controlar la aplicación de la carga (figura 18a). Para medir la carga aplicada, se ocuparon celdas de carga biaxiales de la empresa Metronic con capacidad de 100 toneladas a compresión y 50 a tensión, las cuales fueron conectadas a un equipo de adquisición de datos modelo TDS-300 equipado con sus cajas de conexiones modelo ASW-50C, con capacidad de 50 canales y otra caja ASW-30C con capacidad para 30 canales conectadas en serie para la captura simultánea de datos.

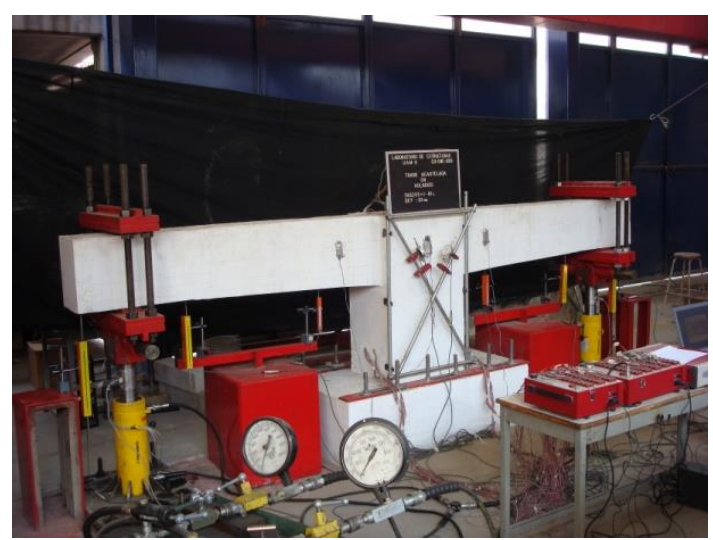

a) Espécimen TASCV3a2-R1c

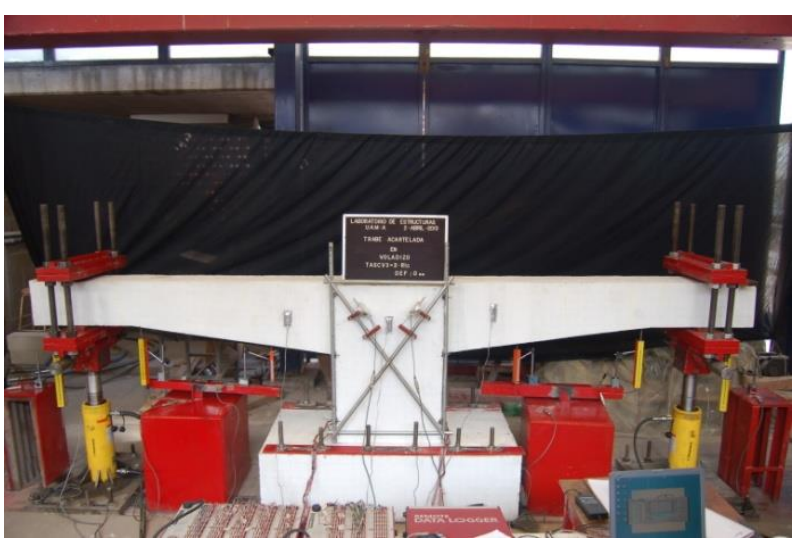

b) Espécimen TASCV3a3-R1c

Figura 18. Dispositivo de prueba utilizado en los ensayes de los especímenes

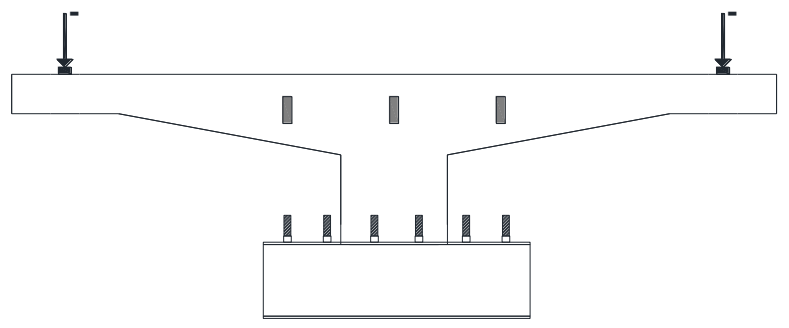

a) Dirección momento negativo

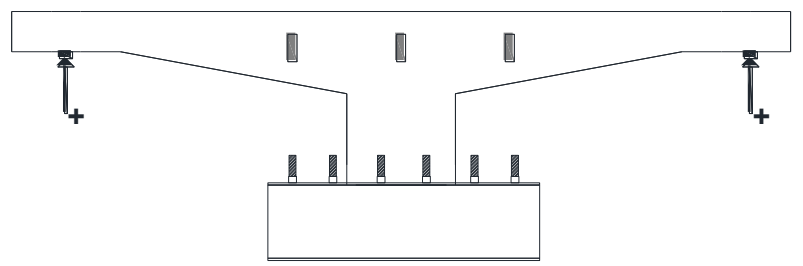

b) Dirección momento positivo

Figura 19. Esquema de la aplicación de la carga cíclica

Las cargas se aplicaron cíclicamente conforme al esquema indicado en la figura 19. Primero se cargó hacia abajo hasta alcanzar un desplazamiento objetivo, lo que corresponde a generar momentos negativos en las cartelas (figura 19a), después se descargó para completar un medio ciclo negativo. Se prosiguió la prueba aplicando carga hacia arriba (figura 19b) hasta alcanzar el mismo desplazamiento 
objetivo, generando momentos positivos en las cartelas; alcanzado ese punto se descargó para completar un medio ciclo positivo y un ciclo completo a la vez.

Los ensayes se controlaron por desplazamientos medidos en el vértice (TI-2 y TD-2, figura 17), en el cambio de sección variable a prismática. Los incrementos de desplazamiento entre cada ciclo fueron de $3 \mathrm{~mm}$ hasta un desplazamiento de $12 \mathrm{~mm}$. Después de alcanzar los $12 \mathrm{~mm}$ de desplazamiento, se incrementaron $4 \mathrm{~mm}$ entre cada ciclo. Para cada sentido de carga $(-\mathrm{P}$ y $+\mathrm{P})$ y cada medio ciclo de carga, se realizó una repetición para evaluar de esa forma la degradación de la rigidez y resistencia del espécimen para un mismo desplazamiento. En la figura 20 se muestra esquemáticamente la historia de carga tipo utilizada en todos los ensayes, donde las deflexiones negativas indican que la carga se aplica hacia abajo (momento negativo y cortante negativo). Debido al daño acumulado en los elementos o en alguna parte de ellos y a la sensibilidad de los transductores de desplazamiento, fue imposible desarrollar rigurosamente la historia de carga mostrada en la figura 20. Los ensayes se terminaron cuando los elementos ya no eran capaces de soportar carga debido al daño presentado. Cabe señalar que los colores mostrados en cada medio ciclo ilustrado en la figura 20 corresponden a los colores con los que se marcaron físicamente las grietas en esos medios ciclos (por ejemplo, figura 25).

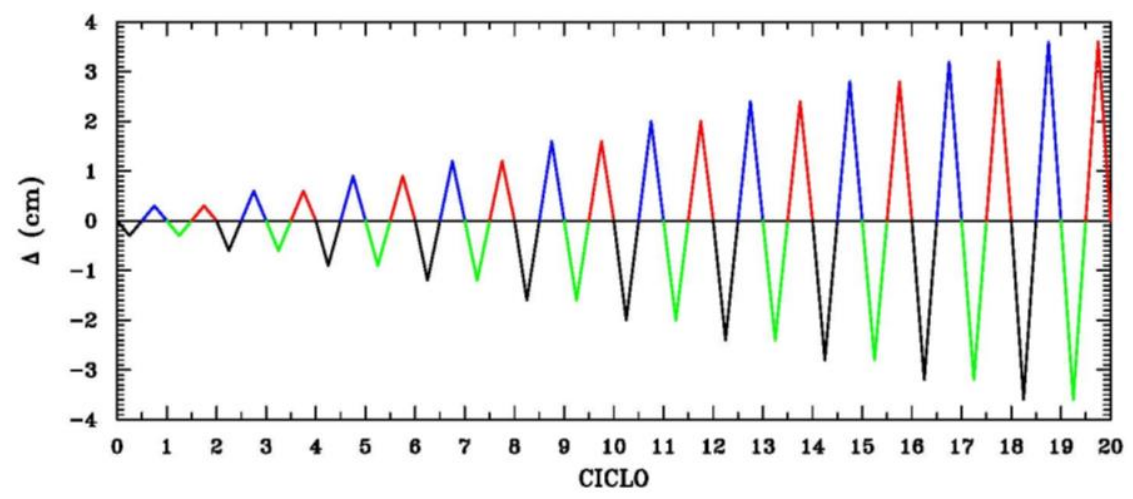

Figura 20. Historia cíclica de desplazamientos aplicada en los especímenes

\section{RESULTADOS EXPERIMENTALES: INSTRUMENTACIÓN EXTERNA}

De los ensayes experimentales se capturaron con el equipo de adquisición de datos las cargas aplicadas, los desplazamientos experimentados y las deformaciones en el acero de refuerzo (longitudinal y transversal) y en el concreto, lo que permitió trazar curvas de histéresis carga-desplazamiento para los distintos puntos del ensaye, así como curvas carga-deformación para cada punto monitoreado con los deformímetros. También se llevó un registro fotográfico y una medición de anchura de grietas máximas durante el progreso de la prueba para cada desplazamiento objetivo (positivo y negativo), y al final de cada par de ciclos a la misma amplitud, lo que permitió posteriormente trazar esquemas del progreso evolutivo de grietas.

A partir de los resultados experimentales se obtuvieron las deflexiones y los cortantes asociados al primer agrietamiento diagonal ( $\Delta_{\text {agr }}$ y $V_{\text {agr }}$ ), último o de falla $\left(\Delta_{\mathrm{u}}\right.$ y $\left.V_{\mathrm{u}}\right)$ y al colapso $\left(\Delta_{\text {clps }}\right.$ y $\left.V_{\text {clps }}\right)$. El primer agrietamiento diagonal se define en función de la aparición de la primera(s) grieta(s) inclinada(s). El estado último o de falla se asoció con la aparición de la primera grieta diagonal frágil, súbita e importante que pone en riesgo la estabilidad del espécimen. El estado de colapso se definió cuando se presentaban desconchamientos y desprendimientos de concreto masivos en la zona de la aplicación de la carga, y en la parte superior e inferior a lo largo de la viga, asociado normalmente a una reducción abrupta de la 
capacidad de carga del espécimen. Estos cortantes y deflexiones características permiten describir el comportamiento de los especímenes.

\section{Análisis de las mediciones de carga y desplazamientos}

Como se aprecia en la figura 17, se eligieron seis puntos de medición de deformación (tres a cada lado). Sin embargo, las lecturas de desplazamiento más representativas son las de los vértices (TI-2 y TD2, figura 17), dado que no sólo sirvieron para monitorear la historia de desplazamientos impuesta, sino que permiten medir la deformación angular (distorsión) de las cartelas. Por ello, en la figura 21 se presentan las curvas de histéresis carga aplicada vs distorsión de la cartela, obtenido como el cociente del desplazamiento en el vértice de la cartela dividido entre la longitud de la cartela $\left(l_{c}=125 \mathrm{~cm}\right.$, figuras 9 a 13), para las cinco trabes ensayadas, la prismática de referencia (TASCV $3 \alpha 0-R 1 c$, figura 21a) y las cuatro acarteladas.

Varios aspectos llaman la atención de las curvas mostradas en esta figura. Quizás lo primero que salta a la vista son las curvas de histéresis de la trabe con mayor ángulo de acartelamiento (figura 21e), que prácticamente no desarrollaron lazos de histéresis importantes, ya que falló prematuramente en el vértice derecho (figura 25), como se discute más adelante. Con excepción de la falla prematura de este espécimen, se aprecia en todos los demás especímenes que la degradación de rigidez y resistencia empieza a ser importante a una distorsión $\Delta$ alrededor de $1.5 \%$. Por otra parte, se confirma que, conforme a la teoría, debido al refuerzo longitudinal asimétrico, la capacidad a cortante es mayor a momento negativo (ciclos negativos) que a momento positivo (ciclos positivos) en la trabes acarteladas (figuras $21 \mathrm{~b}, 21 \mathrm{c} \mathrm{y}$ 21d, tablas 4 y 5). Sin embargo, también lo es para la trabe prismática (figura 21a, tablas 4 y 5), lo que resulta sorpresivo y requiere de un análisis minucioso para determinar a qué se debe exactamente. También se aprecia que las fuerzas cortantes resistidas por las trabes acarteladas no son inferiores a las de la trabe prismática para momento negativo (tablas 4 y 5), con excepción, por supuesto, de la que falló prematuramente en la cartela derecha en el primer ciclo a momento negativo a una amplitud de $1.6 \mathrm{~mm}$, cuya carga se indica en negritas en la tabla 5. Cabe señalar que en las tablas 4 y 5 se marcan con un asterisco (*) las cargas máximas registradas a momento positivo y negativo en las cartelas izquierda y derecha del espécimen TASCV3 $\alpha 4-\mathrm{R} 1 \mathrm{c}$, antes de la falla prematura en su vértice derecho y, por ello, no corresponden exactamente a la carga última de falla, pero se reporta exclusivamente con fines informativos.

Tabla 4. Cortantes experimentales característicos, cartela izquierda

\begin{tabular}{|c|c|c|c|c|c|c|}
\hline Espécimen & $V_{\text {cr }^{-}}(t)$ & $\mathrm{V}_{\mathrm{u}^{-}(\mathrm{t})}$ & $V_{\text {clps }^{-}}(t)$ & $\mathrm{V}_{\mathrm{cr}^{+}}{ }^{+}(\mathrm{t})$ & $\mathrm{V}_{u^{+}}^{+}(t)$ & $\mathrm{V}_{\text {clps }}{ }^{+}(t)$ \\
\hline TASCV3a0-R1c & 11.10 & 29.49 & 19.43 & 10.29 & 21.63 & 15.69 \\
\hline TASCV3a1-R1c & 6.61 & 28.90 & 23.53 & 7.22 & 20.40 & 19.71 \\
\hline TASCV3a2-R1c & 10.75 & 35.06 & 25.82 & 10.16 & 20.07 & 13.51 \\
\hline TASCV3a3-R1c & 10.97 & 30.49 & 24.22 & 10.31 & 21.95 & 20.51 \\
\hline TASCV3a4-R1c & 10.59 & $\begin{array}{c}21.28 * \\
(25.84)^{*}\end{array}$ & $17.61 *$ & 9.97 & $17.93 *$ & - \\
\hline
\end{tabular}

Tabla 5. Cortantes experimentales característicos, cartela derecha

\begin{tabular}{|c|c|c|c|c|c|c|}
\hline Espécimen & $V_{\text {agr }^{-}}(t)$ & $V_{u^{\prime}}(t)$ & $\mathrm{V}_{\text {clps }^{-}(t)}$ & $V_{\mathrm{agr}^{+}}(t)$ & $V_{u^{+}}(t)$ & $\mathrm{V}_{\text {clps }^{+}(t)}$ \\
\hline TASCV3a0-R1c & 12.11 & 30.67 & 18.87 & 10.55 & 21.99 & 20.15 \\
\hline TASCV3a1-R1c & 6.73 & 27.47 & 14.08 & 7.89 & 18.07 & 15.67 \\
\hline TASCV3a2-R1c & 11.32 & 31.05 & 26.11 & 9.37 & 21.61 & 21.61 \\
\hline TASCV3a3-R1c & 10.67 & 30.25 & 25.26 & 9.97 & 22.23 & 20.95 \\
\hline TASCV3a4-R1c & 13.49 & 25.76 & 25.76 & 11.33 & $19.41 *$ & - \\
\hline
\end{tabular}



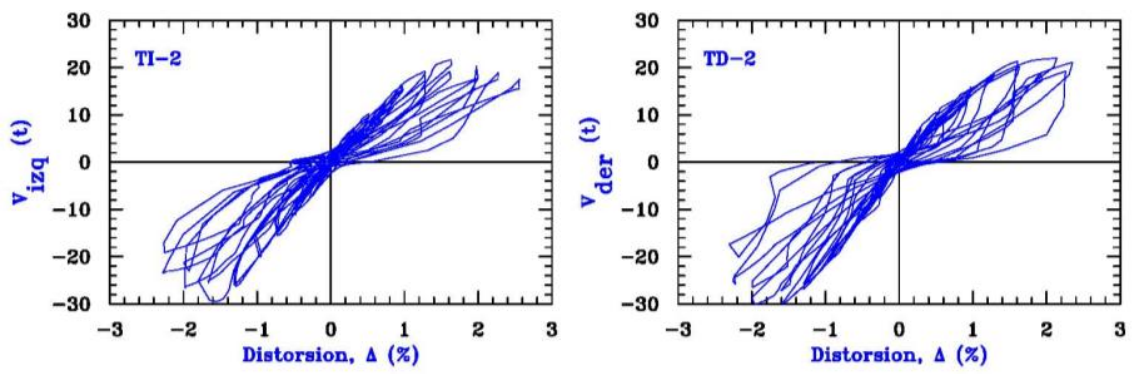

a) $\quad \mathrm{TASCV} 3 \alpha 0-\mathrm{R} 1 \mathrm{c}$

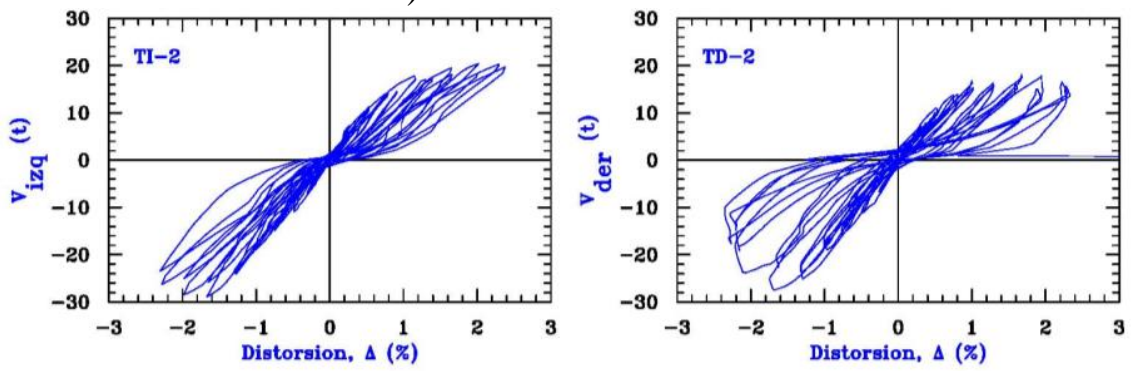

b) TASCV $3 \alpha 1-\mathrm{R} 1 \mathrm{c}$

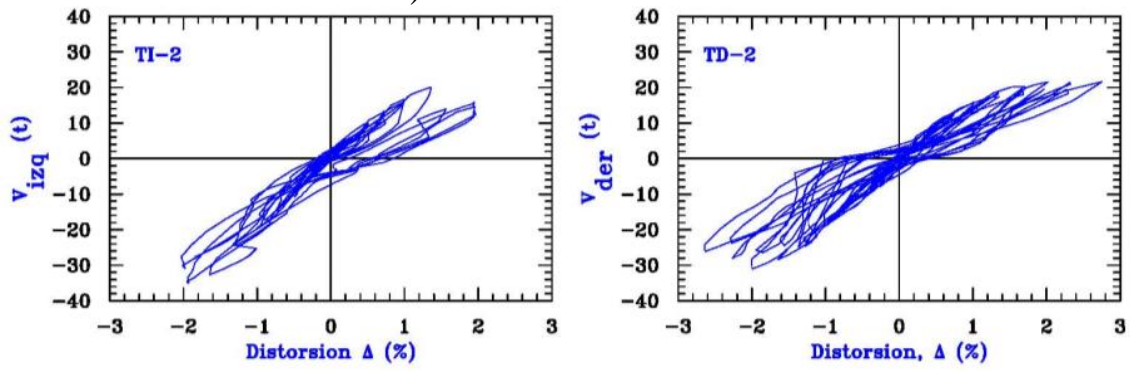

c) TASCV $3 \alpha 2-\mathrm{R} 1 \mathrm{c}$

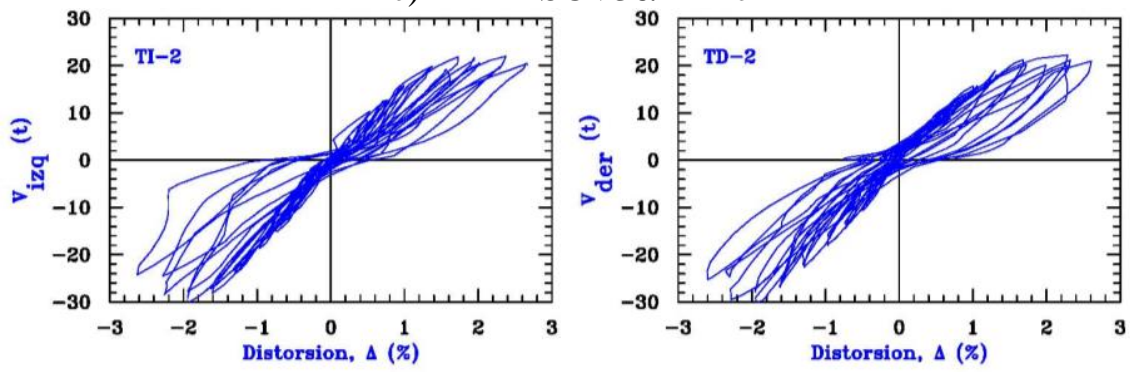

d) TASCV $3 \alpha 3-\mathrm{R} 1 \mathrm{c}$

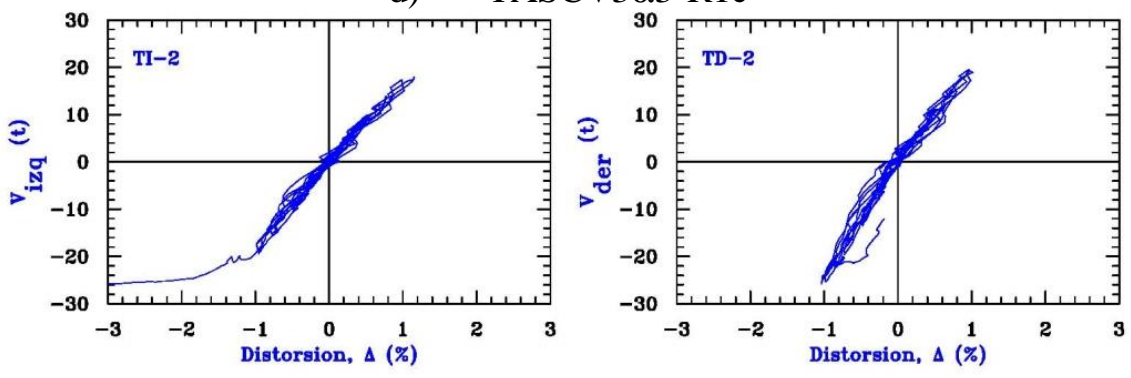

e) TASCV $3 \alpha 4-\mathrm{R} 1 \mathrm{c}$

Figura 21. Curvas de histéresis cortante-distorsión de las cartelas en estudio. Cartelas izquierdas y derechas en el lado izquierdo y derecho, respectivamente 
En general, las resistencias últimas desarrolladas experimentalmente $\left(\mathrm{V}_{\mathrm{u}}^{-} \mathrm{y} \mathrm{V}_{\mathrm{u}^{+}}\right.$, tablas 4 y 5) superan las predichas en el diseño ( $V_{n}$, tabla 2), y la razones de ello se explican con detalle en el artículo compañero (Tena et al. 2017), donde se presentan las interpretaciones de un análisis minucioso hecho con base en los datos experimentales, que permite estimar de manera razonable y racional la aportación a la resistencia por cada concepto (estribos, concreto y refuerzo longitudinal inclinado).

Cabe también señalar que para el espécimen de seis grados (TASCV3 $\alpha 2-R 1 c$, figura 21c) falló súbitamente una parte del dispositivo cuando se estaba aplicando un desplazamiento de $2.0 \mathrm{~cm}(\Delta=$ $1.6 \%$ ), como se explica con mayor detalle en la siguiente sección, y ésto dañó asimétricamente al espécimen y obligó a suspender el ensaye, sustituir la parte dañada del dispositivo y continuarlo después. Es por ello que las curvas mostradas presentan esas irregularidades, pues fue necesario hacer ajustes para obtener las curvas que se presentan. En el espécimen de diez grados (TASCV3 $\alpha 4-R 1$ c, figura 21e) se aprecia que falló el vértice de la cartela derecha (figura 25) y por ello en los lazos de histéresis de la cartela derecha se aprecia la caída abrupta de resistencia. Durante el ensaye se decidió, basado en los ensayes previos, seguir cargando la cartela izquierda, pues se supuso que la carga y deformación últimas estarían ya cercanas a ocurrir. Sin embargo, como se aprecia del lazo de histéresis de la cartela izquierda, este fue un mal juicio y una mala decisión, pues siguió tomando más carga a distorsiones más altas, y es por ello que se ve como la carga crece y se mantiene a altas distorsiones ante una carga que, en ese instante, era aplicada de forma monótona y creciente. Por ello, en las tablas 4 y 6 se indican entre paréntesis y con un asterisco la carga y distorsiones máximas medidas bajo esas circunstancias.

En las tablas 6 y 7 se reportan los desplazamientos máximos en el vértice de las cartelas al momento del primer agrietamiento, la carga última y la de colapso; todos ellos ocurrieron en los primeros medios ciclos de carga. En paréntesis se indican las distorsiones asociadas, expresadas en porcentaje. Los asteriscos tienen el mismo significado que ya se explicó para las resistencias reportadas en las tablas 4 y 5 . Se aprecia que el primer agrietamiento ocurrió generalmente a un desplazamiento cercano a $6 \mathrm{~mm}$ (cercano a $\Delta=0.5 \%$ ) en todos los especímenes, excepto el de tres grados (TASCV3 $\alpha 1-\mathrm{R} 1 \mathrm{c}$ ), donde ocurrió a distorsiones menores. Las trabes acarteladas alcanzaron su carga última a una distorsión $\Delta_{u}$ ligeramente mayor que la de la trabe prismática, particularmente a partir de un ángulo de acartelamiento de 6 grados (especímenes TASCV3 $\alpha 2-R 1$ c y TASCV3 $\alpha 3-R 1 c)$, exceptuando el espécimen TASCV3 $\alpha 4-$ R1c, que falló prematuramente en el vértice de la cartela derecha, como se ha mencionado antes.

Tabla 6. Desplazamientos máximos $(\mathrm{mm})$ en el vértice medidos experimentalmente en la cartela izquierda. Las distorsiones en porcentaje se dan entre paréntesis

\begin{tabular}{|c|c|c|c|c|c|c|}
\hline Espécimen & $\Delta_{\text {agr }^{-}}$ & $\Delta_{u^{-}}$ & $\Delta_{\text {clps }^{-}}$ & $\Delta$ agr $^{+}$ & $\Delta_{\mathrm{u}^{+}}$ & $\Delta$ clps $^{+}$ \\
\hline TASCV3a0-R1c & $5.98(0.48 \%)$ & $19.36(1.55 \%)$ & $32.80(2.62 \%)$ & $6.46(0.52 \%)$ & $20.38(1.63 \%)$ & $32.18(2.57 \%)$ \\
\hline TASCV3a1-R1c & $3.58(0.29 \%)$ & $20.85(1.67 \%)$ & $28.83(2.31 \%)$ & $3.30(0.26 \%)$ & $25.23(2.02 \%)$ & $29.76(2.38 \%)$ \\
\hline TASCV3 $22-R 1 c$ & $6.06(0.48 \%)$ & $24.14(1.93 \%)$ & $32.30(2.58 \%)$ & $6.22(0.50 \%)$ & $16.98(1.36 \%)$ & $33.16(2.65 \%)$ \\
\hline TASCV3a3-R1c & $6.28(0.50 \%)$ & $24.28(1.94 \%)$ & $32.84(2.63 \%)$ & $6.58(0.53 \%)$ & $29.72(2.38 \%)$ & $33.12(2.65 \%)$ \\
\hline TASCV3a4-R1c & $6.36(0.51 \%)$ & $\begin{array}{l}16.17(1.29 \%) \\
41.13(3.29 \%)^{*}\end{array}$ & $46.38(3.71 \%)^{*}$ & $6.69(0.54 \%)$ & $14.43(1.15 \%)^{*}$ & - \\
\hline
\end{tabular}

Tabla 7. Desplazamientos máximos $(\mathrm{mm})$ en el vértice medidos experimentalmente en la cartela derecha. Las distorsiones en porcentaje se dan entre paréntesis

\begin{tabular}{|c|c|c|c|c|c|c|}
\hline Espécimen & $\Delta_{\text {agr }^{-}}$ & $\Delta_{\mathbf{u}^{-}}$ & $\Delta$ clps $^{-}$ & $\Delta \mathrm{agr}^{+}$ & $\Delta_{u^{+}}^{+}$ & $\Delta$ clps $^{+}$ \\
\hline TASCV3a0-R1c & $6.20(0.50 \%)$ & $20.18(1.61 \%)$ & $31.54(2.52 \%)$ & $6.36(0.51 \%)$ & $26.74(2.14 \%)$ & $32.70(2.62 \%)$ \\
\hline TASCV3a1-R1c & $3.24(0.26 \%)$ & $21.04(1.68 \%)$ & $29.18(2.33 \%)$ & $4.62(0.37 \%)$ & $20.90(1.67 \%)$ & $28.48(2.28 \%)$ \\
\hline TASCV3a2-R1c & $6.32(0.51 \%)$ & $24.98(2.00 \%)$ & $32.92(2.63 \%)$ & $6.04(0.48 \%)$ & $25.26(2.02 \%)$ & $34.38(2.75 \%)$ \\
\hline TASCV3a3-R1c & $6.14(0.49 \%)$ & $24.30(1.94 \%)$ & $32.46(2.60 \%)$ & $8.06(0.64 \%)$ & $28.60(2.29 \%)$ & $32.58(2.61 \%)$ \\
\hline TASCV3a4-R1c & $6.03(0.48 \%)$ & $12.96(1.04 \%)$ & $12.96(1.04 \%)$ & $6.33(0.51 \%)$ & $12.72(1.02 \%)^{*}$ & $12.72(1.02 \%)^{*}$ \\
\hline
\end{tabular}




\section{Patrones de agrietamiento}

En las figuras 22 a 24 se presentan los esquemas de agrietamiento registrados para cada uno de los especímenes después de terminar los ciclos aplicados para cada desplazamiento característico $\left(\Delta_{\mathrm{agr}}, \Delta_{\mathrm{u}}\right.$, $\left.\Delta_{\text {clps }}\right)$.
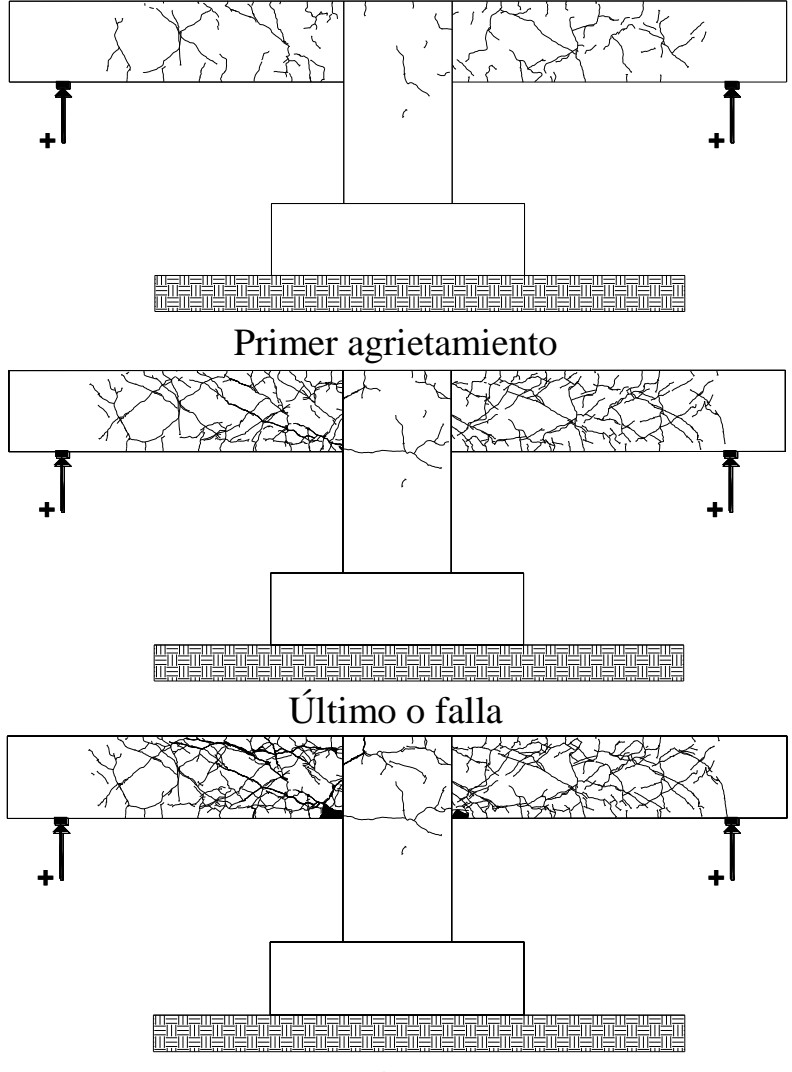

Colapso

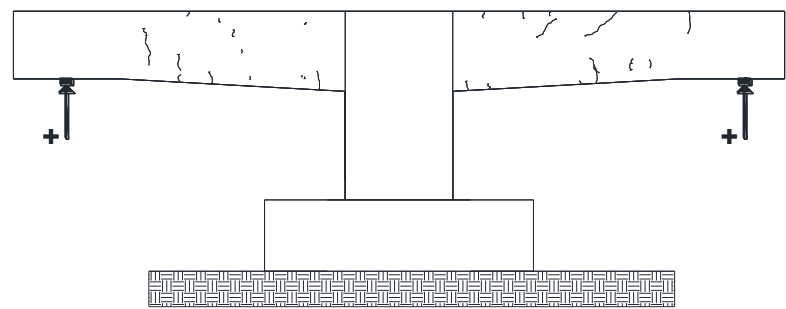

Primer agrietamiento

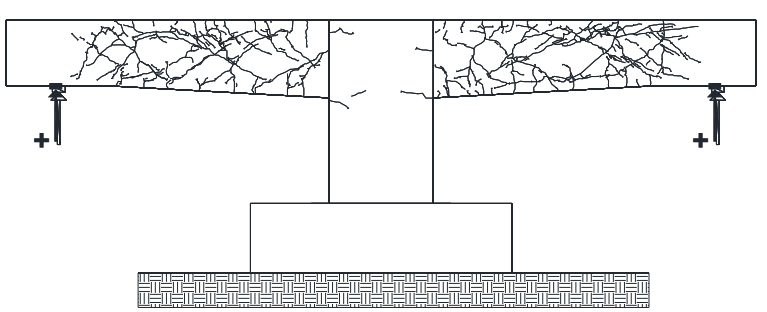

Último o falla

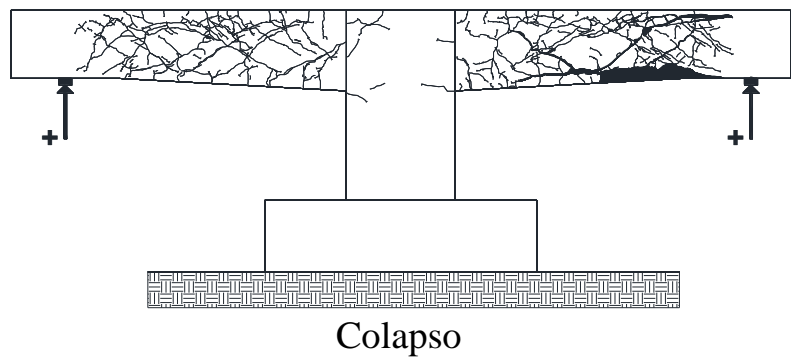

b) Espécimen TASCV3 $\alpha 1-\mathrm{R} 1 \mathrm{c}$

Figura 22. Evolución de patrones de agrietamiento

Para las trabes prismáticas (espécimen TASCV3 $\alpha 0-\mathrm{R} 1 \mathrm{c}$, figura 22a), el primer agrietamiento diagonal sucedió en el segundo medio ciclo de desplazamiento a $\Delta=6 \mathrm{~mm}$; las grietas aparecieron en ambos extremos (izquierdo y derecho). Al finalizar los ciclos correspondientes a $\Delta=12 \mathrm{~mm}$, se observó que el ancho máximo de grieta fue de $0.5 \mathrm{~mm}$ en el lado izquierdo, y en el derecho el ancho máximo de grieta fue $0.25 \mathrm{~mm}$. En el ciclo negativo, correspondiente a un desplazamiento objetivo $\Delta=16 \mathrm{~mm}$, el ancho máximo en la grieta en los lados izquierdo y derecho fue de $0.8 \mathrm{~mm}$. En el segundo ciclo correspondiente al desplazamiento $\Delta=20 \mathrm{~mm}$ se presentó la falla del espécimen en el lado izquierdo $\left(\Delta_{\mathrm{u}}=\right.$ $-19.36 \mathrm{~mm} \mathrm{y} \mathrm{V}_{\mathrm{u}}=-29.49 \mathrm{t}$ ), con un ancho de grieta máximo de $1.5 \mathrm{~mm}$, y se observó desconchamiento cerca del apoyo. Para los siguientes ciclos $(\Delta=28 \mathrm{~mm})$, en el lado izquierdo se observó aplastamiento en la parte inferior del apoyo; además, se observó una abertura de $8 \mathrm{~mm}$ en la zona del vértice y se presentó un desconchamiento generalizado alrededor de la grietas principales, presentando un ancho máximo de grieta de $12 \mathrm{~mm}$. Mientras se estaba descargando, se observó desprendimiento de concreto en el apoyo. El colapso de la trabe ocurrió al término del segundo medio ciclo correspondiente a $\Delta=32 \mathrm{~mm}\left(\Delta_{\text {clps }}=32.8\right.$ $\mathrm{mm}$ y $\mathrm{V}_{\text {clps }}=19.43 \mathrm{t}$ ), ya que se observó perdida de recubrimiento en el lado izquierdo cerca del nudo viga-columna. Además, se observó que mientras se descargaba el espécimen, se seguía perdiendo 
concreto, y se perdió adherencia en el concreto en lecho superior e inferior del refuerzo longitudinal. También se observó que el espécimen ya se encontraba perdiendo capacidad de carga, y fue donde se concluyó el ensaye.

Durante el ensaye experimental de la trabe acartelada TASCV3 $\alpha 1-R 1 c$ (figura 22b), el primer agrietamiento diagonal ocurrió a desplazamientos cercanos a $3 \mathrm{~mm}$ y las grietas aparecieron en ambas cartelas (izquierda y derecha). Al finalizar los ciclos correspondientes a $\Delta=9 \mathrm{~mm}$, se observó que aparecieron más grietas diagonales en ambas cartelas con un ancho de grieta máximo de $0.1 \mathrm{~mm}$. En el medio ciclo negativo correspondiente al desplazamiento objetivo $\Delta=-16 \mathrm{~mm}$, el ancho máximo en la grieta de la cartela del lado derecho era de $1 \mathrm{~mm}$. En los ciclos correspondientes al desplazamiento objetivo $\Delta=20 \mathrm{~mm}$, se presentó la falla del espécimen en la cartela del lado derecho $\left(\Delta_{\mathrm{u}}=-21.04 \mathrm{~mm}\right.$ y $\left.\mathrm{V}_{\mathrm{u}}=-27.47 \mathrm{t}\right)$, con un ancho de grieta máximo de $5 \mathrm{~mm}$. Para los siguientes ciclos $(\Delta=24 \mathrm{~mm})$, se generó una grieta paralela al refuerzo longitudinal y desconchamiento durante la aplicación de la carga en la cartela del lado derecho. El colapso de la trabe ocurrió al término del ciclo correspondiente a un desplazamiento objetivo $\Delta=28 \mathrm{~mm}\left(\Delta_{\text {clps }}=29.18 \mathrm{~mm} \mathrm{y} \mathrm{V}_{\text {clps }}=14.08 \mathrm{t}\right)$, ya que se observó desconchamiento y desprendimiento del recubrimiento en la parte inferior de la cartela derecha; además, se observó desconchamiento en el vértice de la cartela. También se observó que el espécimen ya se encontraba perdiendo capacidad de carga y fue donde se concluyó el ensaye.

Durante el ensaye experimental de la trabe acartelada TASCV3 $\alpha 2-R 1 c$ (figura 23a), el primer agrietamiento diagonal ocurrió en el ciclo a $\Delta=6 \mathrm{~mm}$ y las grietas aparecieron en ambas cartelas. Al finalizar los ciclos correspondientes al desplazamiento objetivo $\Delta=9 \mathrm{~mm}$, se observó que aparecieron más grietas diagonales en ambas cartelas; además, se observó desconchamiento prematuro en el punto de aplicación de la carga en la cartela derecha. En el ciclo negativo correspondiente al desplazamiento objetivo $\Delta=12 \mathrm{~mm}$, el ancho máximo en la grieta de la cartela del lado derecho era de $0.3 \mathrm{~mm}$. Además, se observó ampliación del desconchamiento en el punto de aplicación de la carga en la cartela derecha y se presentó desprendimiento del recubrimiento en la parte inferior del nudo viga-columna, correspondiente a la cartela izquierda y se observó que una grieta atravesó el deformímetro de concreto ubicado en la cartela del lado derecho. En los ciclos correspondientes al desplazamiento objetivo $\Delta=20 \mathrm{~mm}$, se presentó una falla en el dispositivo de carga, el cual se debió al desprendimiento del conector del muñón con la celda de carga. El problema se resolvió colocando un nuevo conector y otra celda de carga. Aunque ésto no perjudicó el ensaye total del espécimen, si se tuvieron que ajustar los datos correspondientes a la celda de carga afectada. En estos ciclos se observó grietas en ambas cartelas, con un ancho de grieta máximo de 0.6 $\mathrm{mm}$ en la cartela de lado izquierdo y de $0.8 \mathrm{~mm}$ en la cartela de lado derecho. En los ciclos correspondientes al desplazamiento objetivo $\Delta=24 \mathrm{~mm}$, se presentó la falla del espécimen en la cartela del lado izquierdo $\left(\Delta_{\mathrm{u}}=-24.14 \mathrm{~mm}\right.$ y $\left.\mathrm{V}_{\mathrm{u}}=-35.06 \mathrm{t}\right)$, con un ancho de grieta máximo de $4 \mathrm{~mm}$; además, se observó desprendimiento del recubrimiento en la cara posterior de cartela izquierda. El colapso de la trabe ocurrió al término del medio ciclo correspondiente al desplazamiento objetivo $\Delta=32 \mathrm{~mm}$, ya que se observó desprendimiento del recubrimiento en la parte superior e inferior de la cartela izquierda. También se observó que el espécimen ya se encontraba perdiendo capacidad de carga, y fue donde se concluyó el ensaye.

Durante el ensaye experimental de la trabe acartelada TASCV3 $\alpha 3-R 1 c$ (figura 23b), el primer agrietamiento diagonal ocurrió en el tercer medio ciclo del desplazamiento objetivo $\Delta=6 \mathrm{~mm}$, y las grietas aparecieron en ambas cartelas. Al finalizar los ciclos correspondientes al desplazamiento objetivo $\Delta=12 \mathrm{~mm}$, se observó que las grietas del ciclo anterior se extendieron y aparecieron grietas en el vértice de ambas cartelas. En el primer ciclo negativo correspondiente al desplazamiento objetivo $\Delta=16 \mathrm{~mm}$, el ancho máximo en la grieta de la cartela del lado izquierdo era de $0.5 \mathrm{~mm}$. Además, se observó 
desconchamiento y desprendimiento en el punto de aplicación de la carga en la cartela izquierda. En la cartela de lado derecho, el ancho máximo de grieta fue de $0.15 \mathrm{~mm}$. Además, se presentó desprendimiento del soporte del marco de los transductores tipo reloj en la parte superior del nudo viga-columna. En el primer ciclo correspondiente al desplazamiento objetivo $\Delta=24 \mathrm{~mm}$, se presentó la falla del espécimen en la cartela del lado izquierdo $\left(\Delta_{\mathrm{u}}=-24.28 \mathrm{~mm} \mathrm{y} \mathrm{V}_{\mathrm{u}}=-30.49 \mathrm{t}\right)$, con un ancho de grieta máximo de $4 \mathrm{~mm}$. Para los siguientes ciclos, a un desplazamiento objetivo $\Delta=28 \mathrm{~mm}$, en la cartela del lado izquierdo se observó desconchamiento en la zona del vértice y en el punto de aplicación de carga, mientras que en la parte posterior de la cartela de lado derecho se observó un ancho de grieta máximo de $7 \mathrm{~mm}$; además, se presentó desconchamiento cercano al punto de aplicación de la carga mientras se estaba descargando el espécimen. El colapso de la trabe ocurrió al término del medio ciclo positivo correspondiente a un desplazamiento objetivo $\Delta=32 \mathrm{~mm}$, ya que se observó desprendimiento de concreto en la parte central de la cartela izquierda. Además, se observó desconchamiento en el punto de aplicación de carga, y se perdió adherencia en el concreto en lecho superior del refuerzo longitudinal. También se observó que el espécimen ya se encontraba perdiendo capacidad de carga, y fue donde se concluyó el ensaye.

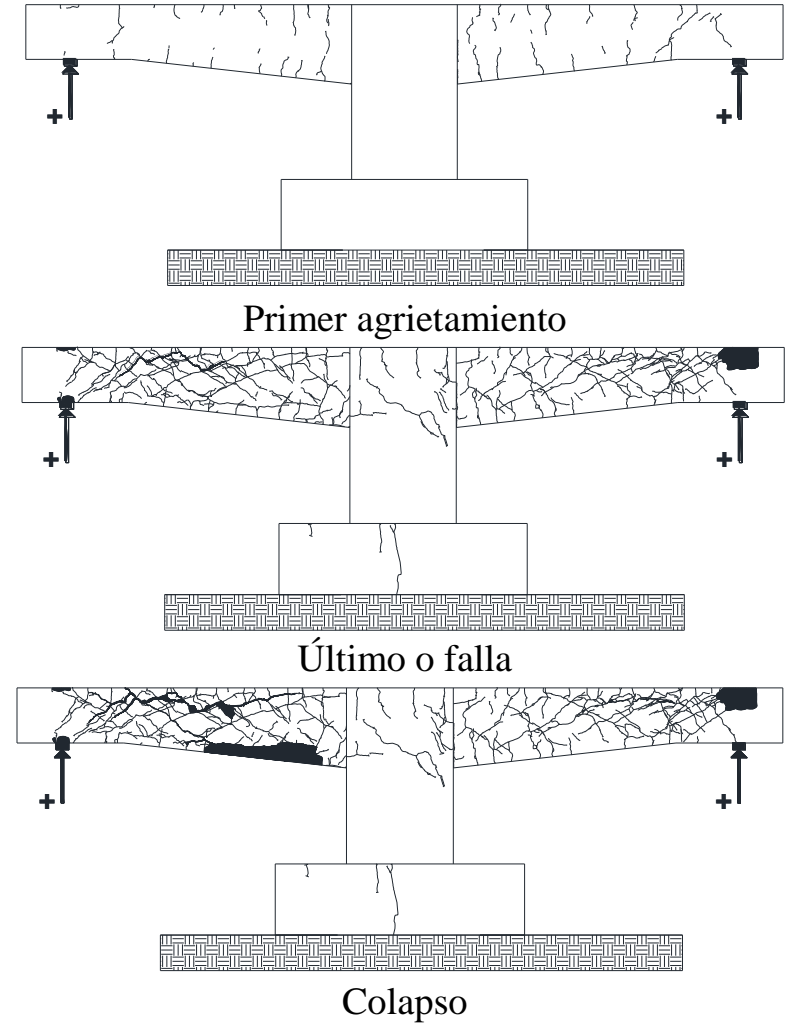

a) Espécimen TASCV3 $\alpha 2-\mathrm{R} 1 \mathrm{c}$

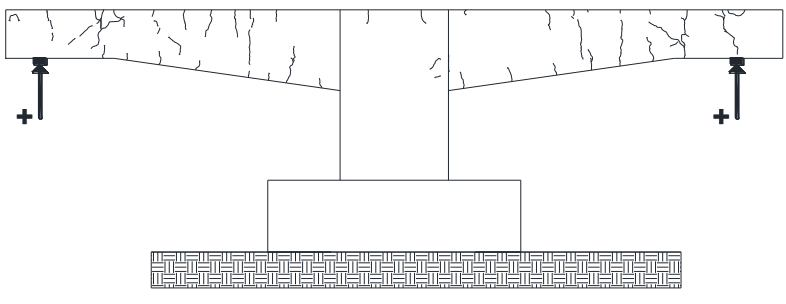

Primer agrietamiento
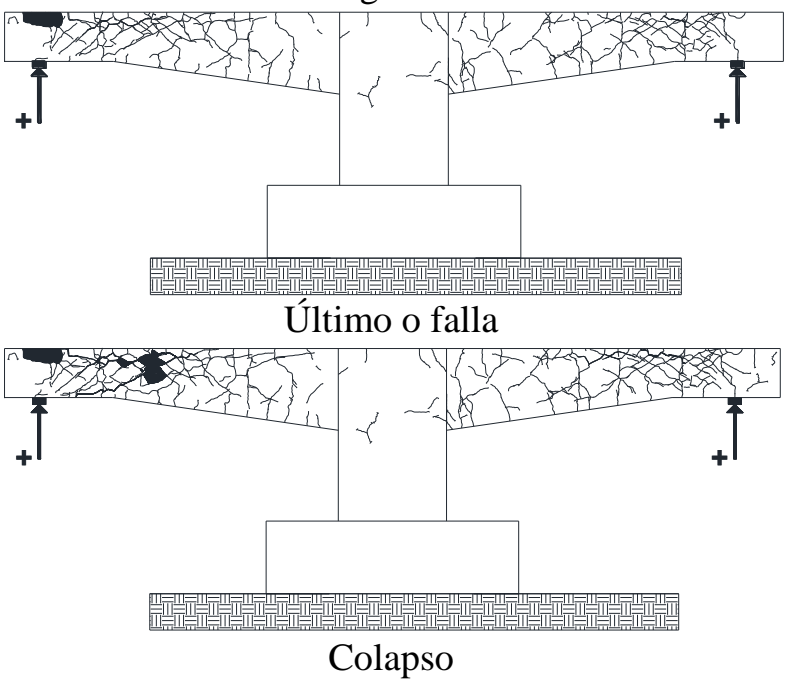

b) Espécimen TASCV3 $\alpha 3-\mathrm{R} 1 \mathrm{c}$

Figura 23. Evolución de patrones de agrietamiento

Durante el ensaye experimental de la trabe acartelada TASCV3 $\alpha 4-R 1 c$ (figura 24), el primer agrietamiento diagonal ocurrió en el tercer medio ciclo del desplazamiento objetivo $\Delta=6 \mathrm{~mm}$, y las grietas aparecieron en ambas cartelas. Al finalizar los ciclos correspondientes al desplazamiento objetivo $\Delta=9 \mathrm{~mm}$, la anchura de las grietas mayores por cortante eran de $0.2 \mathrm{~mm}$ en la cartela izquierda y de 0.6 $\mathrm{mm}$ en la cartela derecha. Durante el primer ciclo al desplazamiento objetivo $\Delta=-12 \mathrm{~mm}$ se presentó un ligero desconchamiento en el recubrimiento de la cartela del lado izquierdo. En el segundo ciclo a ese desplazamiento objetivo $\Delta=-12 \mathrm{~mm}$ la anchura de la grieta mayor creció a $1.5 \mathrm{~mm}$ en la cartela derecha, 
y al cerrar los segundos ciclos a un desplazamiento objetivo $\Delta=+12 \mathrm{~mm}$ la anchura de la grieta principal a momento positivo era de $4.0 \mathrm{~mm}$. Esto anticipaba lo que ocurrió en el primer ciclo a momento negativo para el desplazamiento objetivo $\Delta=-16 \mathrm{~mm}$ : la falla prematura por cortante de la cartela derecha en el vértice, como se aprecia más claramente en la figura 25. Dado que los patrones de agrietamientos en ambas cartelas eran similares antes de esta falla abrupta, y con base en lo observado en ensayes previos, se intuyó que la falla en la cartela izquierda estaba cercana. Esta hipótesis la reforzaba el hecho que, por las restricciones del dispositivo experimental (peso del espécimen y características de la grúa), el máximo peralte de las trabes estaba limitado a $45 \mathrm{~cm}$, y para poder estudiar un ángulo cercano a 10 grados, se tuvo que tener en el vértice una sección transversal de $23 \mathrm{~cm}$ de peralte y $25 \mathrm{~cm}$ de ancho, que no es la más adecuada y, muy seguramente, esto explica en gran medida por qué se presentó esta falla abrupta en uno de los vértices (posteriormente se determinó que la resistencia del concreto a la ruptura era muy baja, $f_{r}=$ $19.3 \mathrm{~kg} / \mathrm{cm}^{2}$, y esto es también un factor que influyó). Por estas razones se decidió seguir cargando la cartela izquierda, y como ya se mencionó anteriormente, al final de cuentas resultó ser una mala decisión. Así sucede a veces en los experimentos cuando se presenta algo inesperado, se tienen que tomar decisiones que a veces pueden no ser las mejores. Este equipo de trabajo es honesto y las cuenta como experiencia que puede serle útil a otros, más que mañosamente "cortar" o no presentar estos datos de este espécimen, que sería lo más fácil (pero deshonesto).

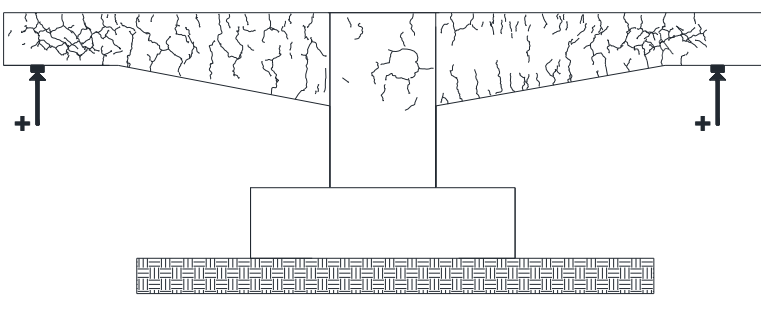

Primer agrietamiento

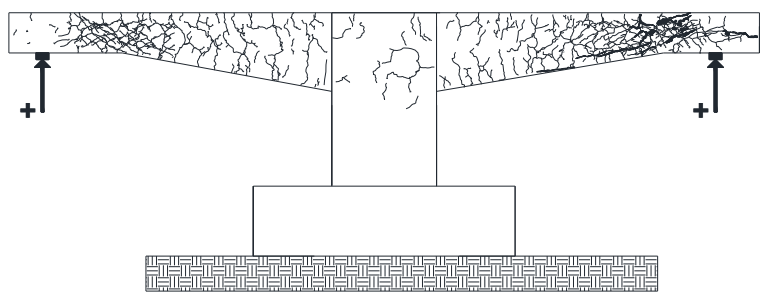

Último o falla (colapso cartela derecha)

Figura 24. Evolución de patrones de agrietamiento para el espécimen TASCV3 $\alpha 4-R 1 c$

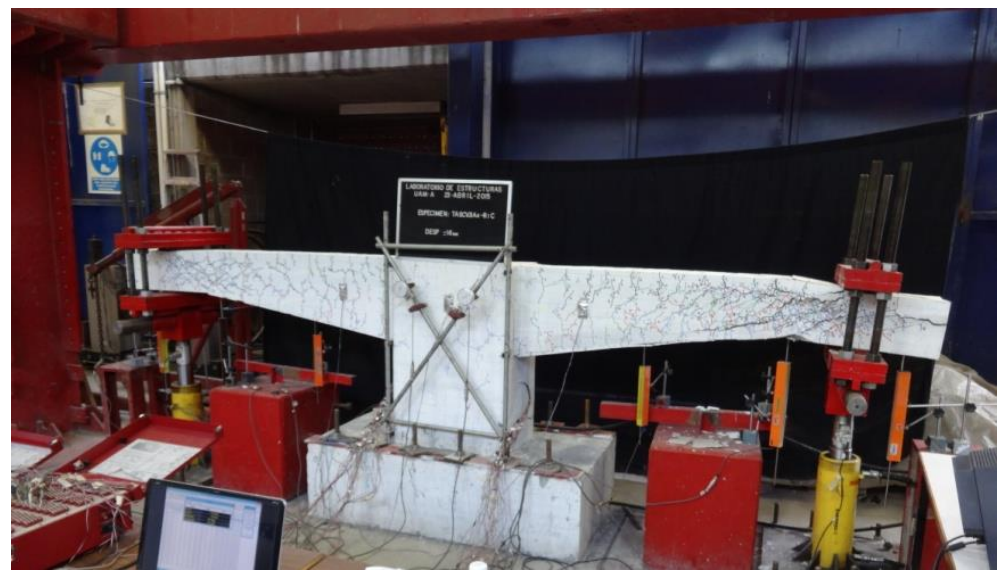

Figura 25. Falla en el vértice de la cartela derecha del espécimen TASCV3 $\alpha 4-R 1 c$

Entonces, haciendo a un lado los resultados obtenidos para el espécimenTASCV3 $\alpha 4-R 1 c$ (figuras 24 y 25), de los patrones de agrietamiento mostrados en las figuras 22 y 23 , se comprueba lo observado en ensayes anteriores ante cargas estáticas y cíclicas: las trabes acarteladas distribuyen de mejor manera el daño al desarrollar más grietas y de menor espesor que las trabes prismáticas, lo cual es más notorio a 
medida que aumenta el ángulo de acartelamiento (comparar, por ejemplo, los patrones de las figuras 22a con los de las figuras 23a y $23 b$ ).

\section{Degradación de la resistencia}

Para ilustrar más claramente cómo se degrada la resistencia a cortante de los especímenes conforme se aumenta el nivel de deformación, y el impacto que tiene la repetición de ciclos de carga a la misma amplitud, se trazaron envolventes de las respuestas máximas de las curvas carga-deformación para cada ciclo (primeros y segundos) para cada trabe en estudio, los cuales se presentan en la figura 26. En la figura 26 se aprecian claramente dos aspectos: 1) como era de esperarse, la degradación de resistencia a cortante comienza a partir de que se alcanza $\mathrm{V}_{\mathrm{u}}$ (tablas 4 y 5), y la caída en la curva es más pronunciada en las trabes prismáticas que en las acarteladas y, 2) la degradación en la resistencia de los segundos ciclos con respecto a los primeros es mayor y más notoria después de $\mathrm{V}_{\mathrm{u}}$. Antes de $\mathrm{V}_{\mathrm{u}}$, la diferencia entre las envolventes de los primeros y segundos ciclos es poco significativa.

Cabe señalar que en la figura 26e se traza con línea punteada los datos de la carga monótonamente aplicada después de la falla en la cartela del espécimen TASCV3 $\alpha 4-R 1 c$, como se ha explicado anteriormente.

\section{Degradación de la rigidez}

La rigidez es un parámetro que depende tanto de la geometría, como de las propiedades mecánicas de los materiales con los que se han construido un elemento o estructura. La degradación de rigidez en los especímenes sometidos ante carga cíclica se evaluó utilizando la rigidez de ciclo $\left(K_{c}\right)$, definida como la pendiente de la recta secante que une a los picos de dos medios ciclos durante un ciclo completo de carga calculada como:

$$
K_{c}=\frac{V^{+}+V^{-} \mid}{\Delta^{+}+\Delta^{-}}
$$

donde $V^{+}$es la carga máxima del medio ciclo positivo, $V^{-}$es la carga máxima del medio ciclo negativo, $\Delta^{+}$ es la deflexión asociada a $V^{+}$y $\Delta^{-}$es la deflexión asociada a $V^{-}$.

En la figura 27 se muestra la evolución de la rigidez para los primeros ciclos y los de repetición; estas rigideces están normalizadas por la rigidez elástica inicial de los ciclos $K_{E L}$, la cual es la rigidez máxima de los ciclos de histéresis hasta antes del primer agrietamiento diagonal. En la tabla 8 se indica el valor de la rigidez inicial para cada trabe en estudio, así como los valores de las rigideces normalizadas a la carga de agrietamiento $\left(\mathrm{K}_{\mathrm{agr}}\right)$, a la falla $\left(\mathrm{K}_{\mathrm{u}}\right)$ y al colapso $\left(\mathrm{K}_{\mathrm{clps}}\right)$. También se reporta en la tabla 8 el módulo de elasticidad $E_{c}$ obtenido de cilindros probados a la edad del ensaye de los especímenes. Se observa de la tabla 8 que la rigidez elástica en ambas cartelas del espécimen no es idéntica, a pesar que la geometría si lo es y que el colado se realizó monolíticamente, pero no los valores de $E_{c}$. Esto es más notorio en la trabe prismática (TASCV $3 \alpha 0-\mathrm{R} 1 \mathrm{c}$ ) y en la trabe con el mayor ángulo de acartelamiento, TASCV $3 \alpha 4-R 1 c$. Este hecho confirma que en las estructuras de concreto reforzado sus propiedades varían notablemente (entre ellas, $E_{c}$ ), aún en estructuras totalmente simétricas. 

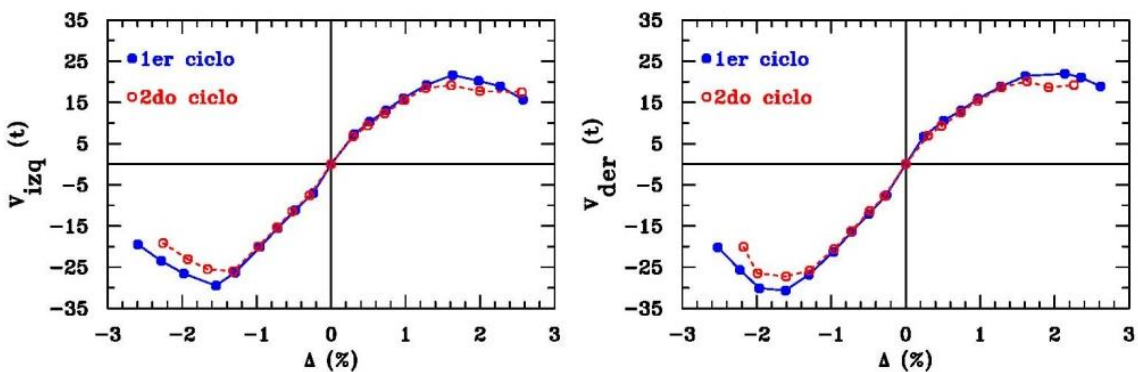

a) $\quad$ TASCV $3 \alpha 0-R 1 c$

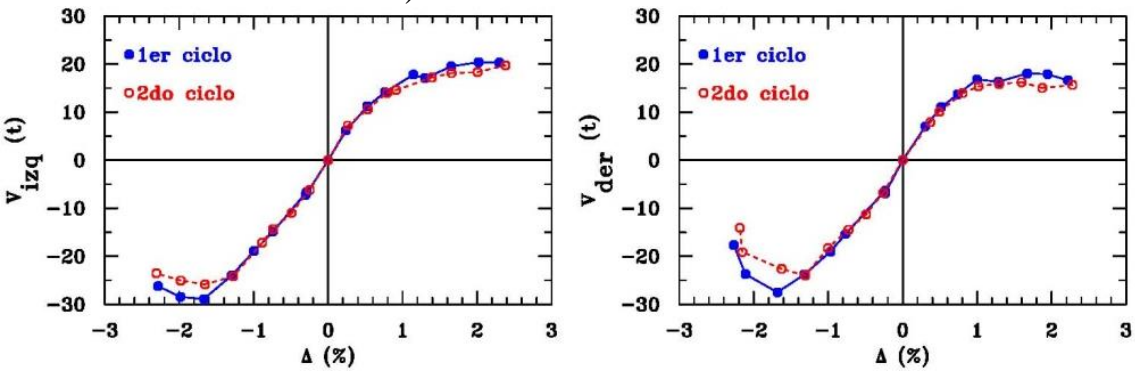

b) TASCV $3 \alpha 1-\mathrm{R} 1 \mathrm{c}$
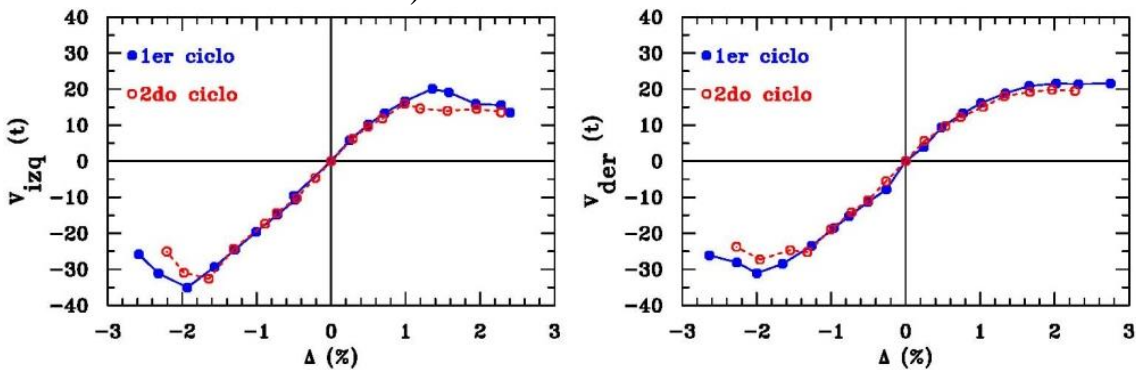

c) TASCV3 $22-\mathrm{R} 1 \mathrm{c}$
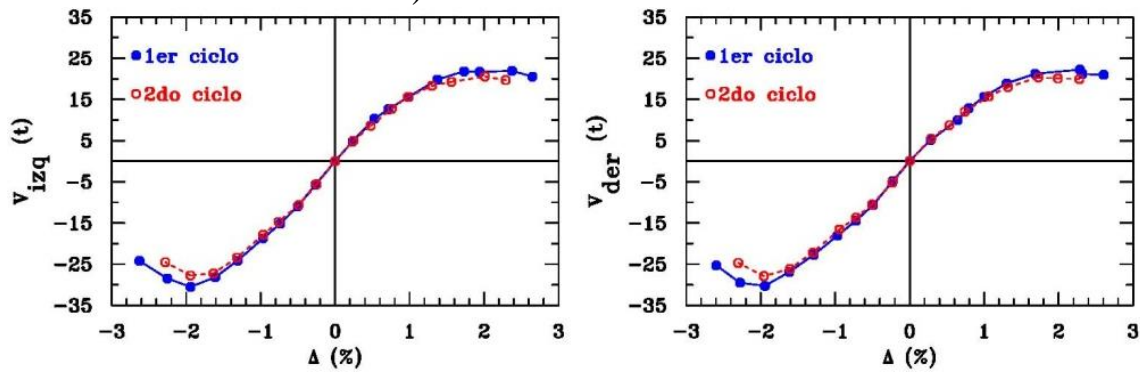

d) TASCV3 $\alpha 3-\mathrm{R} 1 \mathrm{c}$
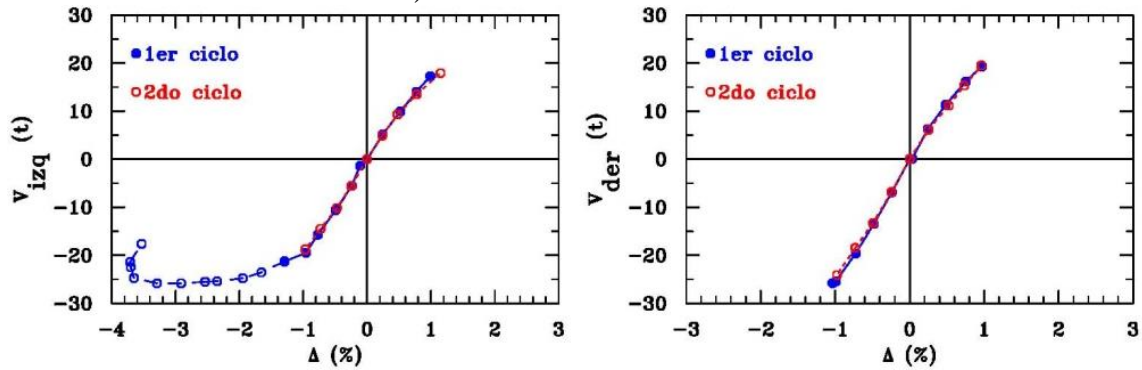

e) TASCV3 $\alpha 4-\mathrm{R} 1 \mathrm{c}$

Figura 26. Envolventes de respuestas máximas de los especímenes en estudio. Cartelas izquierdas y derechas en el lado izquierdo y derecho, respectivamente 

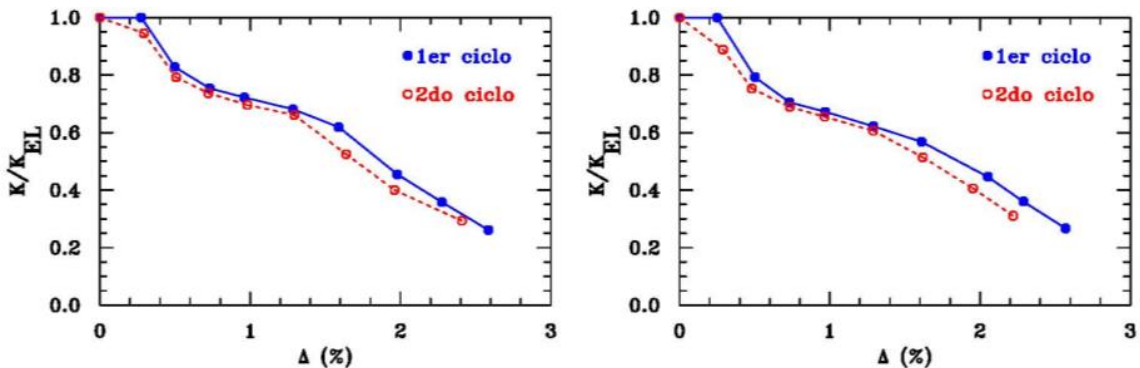

a) TASCV3 $\alpha 0-\mathrm{R} 1 \mathrm{c}$
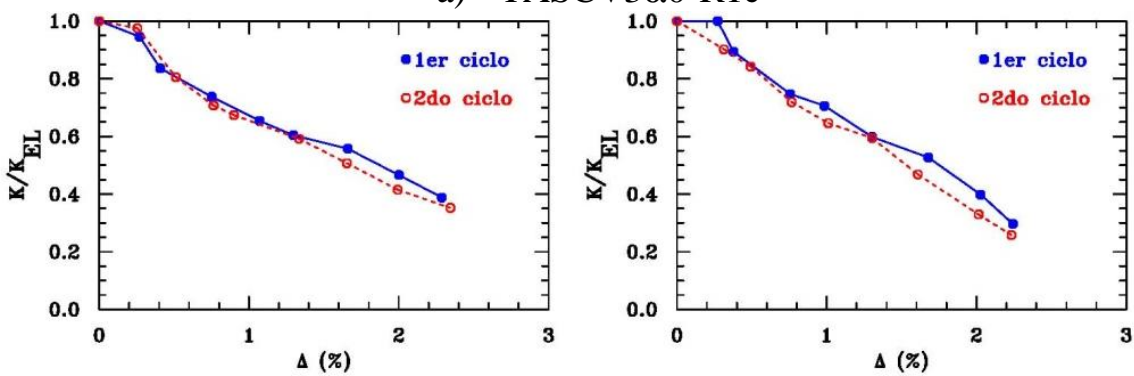

b) TASCV $3 \alpha 1-\mathrm{R} 1 \mathrm{c}$
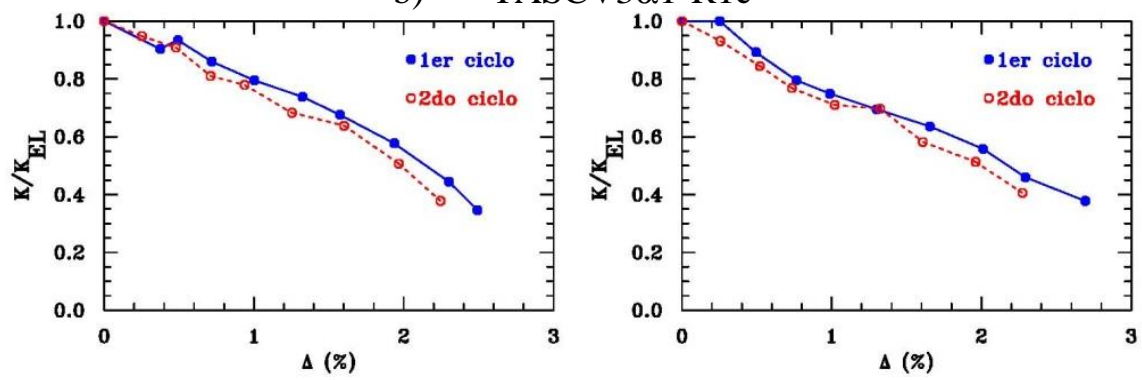

c) TASCV3 $22-\mathrm{R} 1 \mathrm{c}$
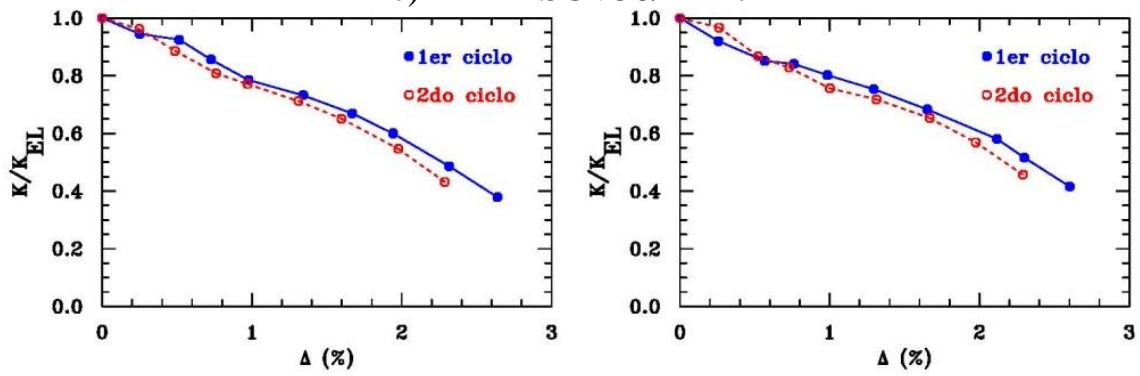

d) TASCV3 $\alpha 3-\mathrm{R} 1 \mathrm{c}$
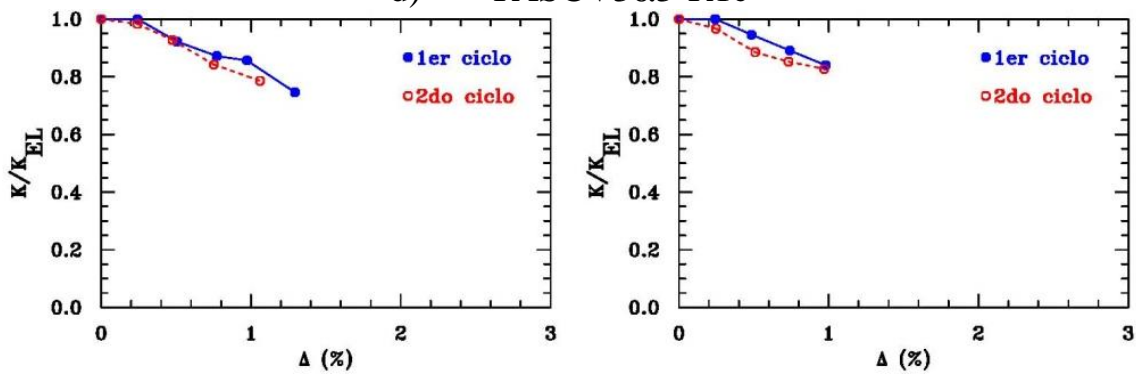

e) TASCV3 $\alpha 3-\mathrm{R} 1 \mathrm{c}$

Figura 27. Degradación de rigidez de los especímenes en estudio. Cartelas izquierdas y derechas en el lado izquierdo y derecho, respectivamente 
Tabla 8. Degradación de rigidez calculada a partir de las curvas de histéresis de las cartelas

\begin{tabular}{|c|c|c|c|c|c|c|c|c|c|c|}
\hline \multirow[t]{2}{*}{ Espécimen } & \multicolumn{5}{|c|}{ Cartela izquierda } & \multicolumn{5}{|c|}{ Cartela derecha } \\
\hline & $\begin{array}{c}\mathbf{E}_{\mathrm{c}} \\
\left(\mathrm{kg} / \mathrm{cm}^{2}\right)\end{array}$ & $\mathbf{K}_{\mathrm{EL}}(\mathbf{t} / \mathbf{m m})$ & $\mathbf{K}_{\mathrm{agr}} / \mathbf{K}_{\mathrm{EL}}$ & $\mathbf{K}_{\mathrm{u}} / \mathbf{K}_{\mathrm{EL}}$ & $\overline{\mathbf{K}_{\text {clps }} / \mathbf{K}_{\mathrm{EL}}}$ & $\begin{array}{c}\mathbf{E}_{\mathrm{c}} \\
\left(\mathrm{kg} / \mathrm{cm}^{2}\right)\end{array}$ & $\mathbf{K}_{\mathrm{EL}}(\mathbf{t} / \mathbf{m m})$ & $\mathbf{K}_{\mathrm{agr}} / \mathbf{K}_{\mathrm{EL}}$ & $\mathbf{K}_{\mathbf{u}} / \mathbf{K}_{\mathrm{EL}}$ & $\mathbf{K}_{\text {clps }} / \mathbf{K}_{\mathbf{E L}}$ \\
\hline TASCV3a0-R1c & 125,685 & 2.08 & 0.83 & 0.62 & 0.26 & 128,891 & 2.28 & 0.79 & 0.57 & 0.27 \\
\hline TASCV3a1-R1c & 130,628 & 2.15 & 0.94 & 0.56 & 0.39 & 123,893 & 2.06 & 0.90 & 0.53 & 0.30 \\
\hline TASCV3a2-R1c & 119,648 & 1.82 & 0.93 & 0.58 & 0.35 & 117,833 & 1.88 & 0.89 & 0.56 & 0.38 \\
\hline TASCV3a3-R1c & 124,279 & 1.79 & 0.93 & 0.60 & 0.38 & 120,967 & 1.71 & 0.85 & 0.52 & 0.42 \\
\hline TASCV3a4-R1c & 122,525 & 1.76 & 0.92 & $0.75 *$ & - & 124,604 & 2.17 & 0.95 & $0.83^{*}$ & $0.83^{*}$ \\
\hline
\end{tabular}

En cuanto a las curvas de degradación de rigidez, se aprecia que aunque en los primeros ciclos y los de repetición siguen una misma tendencia, es más notoria la degradación de rigidez en los ciclos de repetición, principalmente a partir del primer agrietamiento diagonal (figura 27). Este fenómeno se asocia al agrietamiento del concreto, el cual al no tener una plasticidad perfecta, pierde sus propiedades cuando éste se presenta. También es muy importante señalar que de acuerdo con los datos reportados en la tabla 8, al primer agrietamiento, la máxima pérdida de rigidez fue del $21 \%$ en la cartela derecha del espécimen TASCV $3 \alpha 0-R 1 c$, el cual ya mostraba un agrietamiento significativo antes del primer agrietamiento por cortante (figura 22a). La rigidez efectiva a la carga última es casualmente ligeramente mayor al $50 \%$ para todos los especímenes.

Si uno observa los patrones de agrietamiento correspondientes al estado último o de falla de los especímenes (figuras 22 a 24), se aprecia que este daño es muy extenso y fácilmente observable. Por ello, nos parece pertinente reiterar nuestro desacuerdo con respecto a que en muchas normas internacionales de concreto, incluidas el ACI-318 y las NTCC-04, para fines de diseño soliciten considerar secciones "agrietadas" del $50 \%$ en vigas que fallan a flexión, y esa rigidez secante la consideren como una "elástica equivalente" para fines de diseño, argumentando que esa "pérdida de rigidez" está asociado al agrietamiento por contracción del concreto, deslizamientos de barras, etcétera (de hecho, todos fenómenos no lineales). Los miembros de esos comités mejor deberían reconocer que si en muchas ocasiones la rigidez elástica que estiman analíticamente con una función cuadrática en función de $f_{c}^{\prime}$ no coincide con las rigideces iniciales experimentales, se debe a que calculan el módulo de elasticidad conforme a la ecuación que sugiere esa norma con base en la resistencia medida en los cilindros, como si esta propiedad fuera una constante absoluta y no una variable que depende de muchísimos factores. De hecho, $E_{c}$ de los especímenes (tabla 8) varían entre $6,784 \sqrt{f^{\prime}{ }_{c}}$ y $7,963 \sqrt{f^{\prime}}$, muy por debajo (entre $48 \%$ y $57 \%$ ) de la ecuación propuesta por las NTCC-04 $\left(E_{c}=14,000 \sqrt{f_{c}^{\prime}}\right)$. Seguramente la incertidumbre en la estimación del módulo de elasticidad es más responsable de que no coincidan las rigideces elásticas teóricas con las iniciales experimentales, que unas cuantas y pequeñas fisuras por contracción, no siempre apreciables a simple vista, y que se ha demostrado con elementos finitos que no serían responsables de una pérdida inicial de rigidez de ese tamaño (Archundia et al. 2005). El agrietamiento por deslizamiento de las barras longitudinales se observa a grandes distorsiones, generalmente cercanas a la carga última (figs. 22 a 24).

\section{Disipación de energía}

Se calculó la energía histerética disipada por cada ciclo, $E_{h}$, y se separó el acumulado de los primeros ciclos de carga a un desplazamiento objetivo de los segundos ciclos a ese desplazamiento objetivo (o ciclos de repetición). En la figura 28 se presenta la energía histerética acumulada en los primeros y segundos ciclos para cada uno de los especímenes en estudio. En dicha figura se observa que, salvo el elemento prismático (TASCV $3 \alpha 0-\mathrm{R} 1 \mathrm{c}$ ) en su extremo derecho y la trabe acartelada TASCV $3 \alpha 3-$ $\mathrm{R} 1 \mathrm{c}$ en su cartela izquierda, la energía en los ciclos primeros y de repetición es prácticamente la misma en ambas cartelas, hasta la aparición del primer agrietamiento diagonal (ciclo 2). 

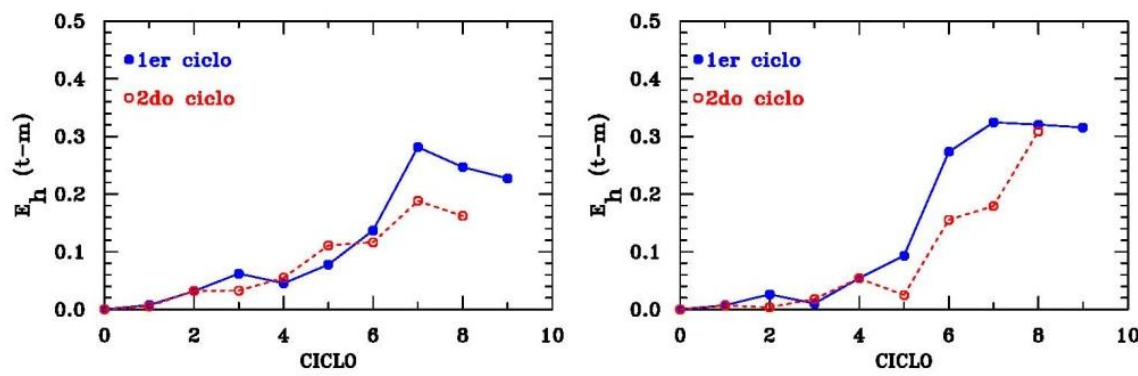

a) TASCV3 $\alpha 0-\mathrm{R} 1 \mathrm{c}$
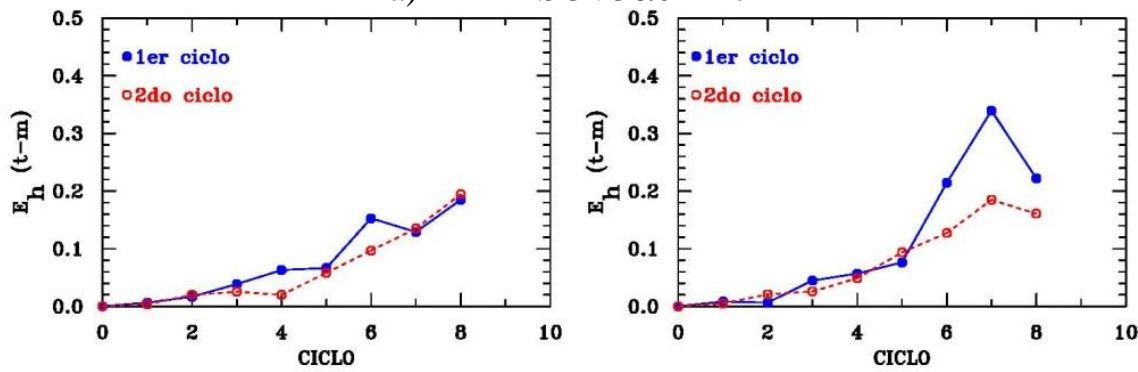

b) TASCV3 $\alpha 1-\mathrm{R} 1 \mathrm{c}$
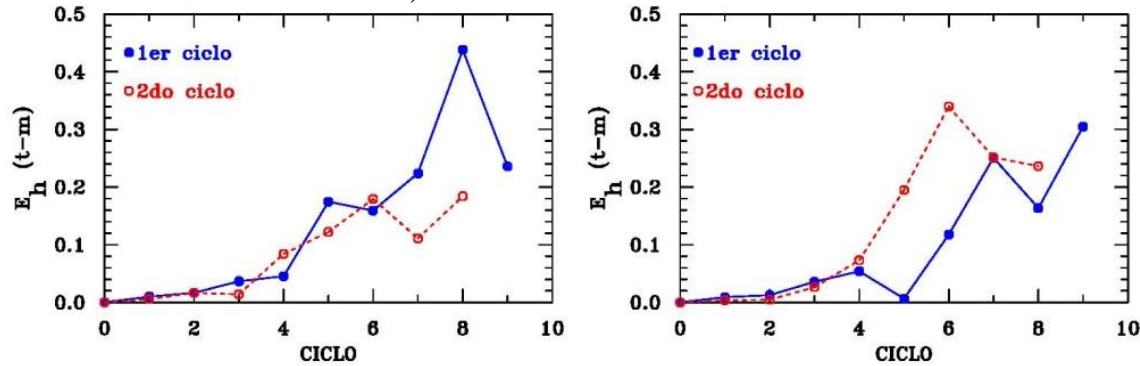

c) TASCV3 $\alpha 2-\mathrm{R} 1 \mathrm{c}$
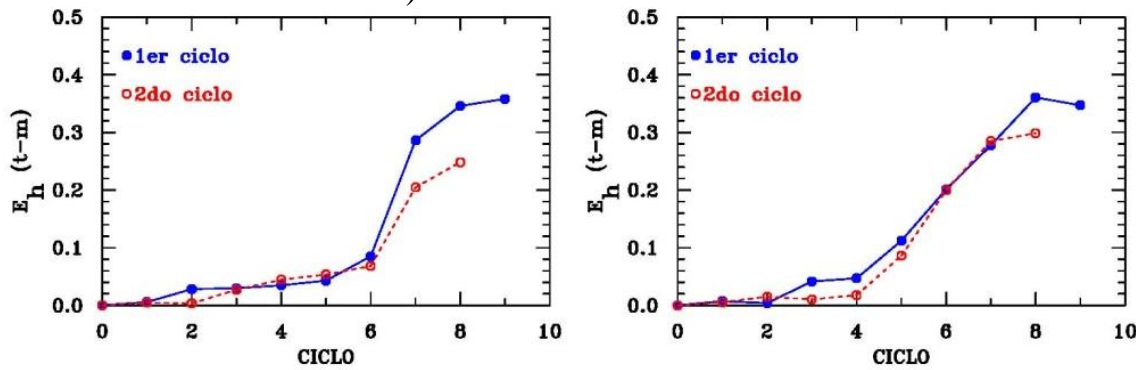

d) TASCV3 $23-\mathrm{R} 1 \mathrm{c}$
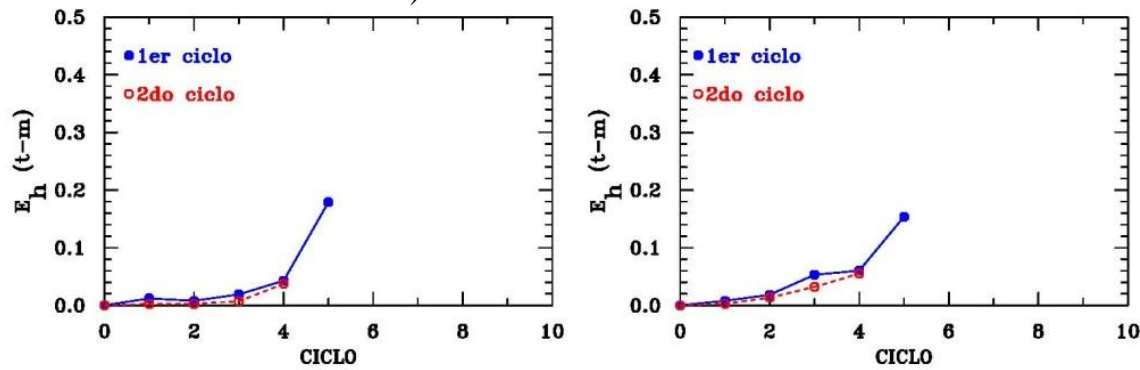

e) TASCV3 $\alpha 4-\mathrm{R} 1 \mathrm{c}$

Figura 28. Energía histerética disipada acumulada, $E_{h}$, de los especímenes en estudio. Cartelas izquierdas y derechas en el lado izquierdo y derecho, respectivamente 
Una vez presentado el primer agrietamiento diagonal, y hasta poco antes de la falla, la tendencia observada es que normalmente la energía disipada para alcanzar con un desplazamiento objetivo es mayor en los primeros ciclos que en los de repetición, con algunas excepciones en algunos ciclos específicos. Este comportamiento se explica con lo observado en los experimentos, ya que las grietas principales y las nuevas grietas se generaron normalmente en los primeros ciclos, mientras que durante los ciclos de repetición generalmente no se generaron nuevas grietas de importancia, sino que principalmente se ensanchaban o se extendían las grietas ya generadas. También es notorio que en algunos elementos se generó un súbito incremento de energía en el instante en que se formó la grieta de falla (figura 28).

En la figura 29 se presenta la energía histerética acumulada (primeros y segundos ciclos) para los especímenes en estudio, donde se aprecia que, en general, las trabes acarteladas disipan una cantidad similar de energía que las trabes prismáticas.
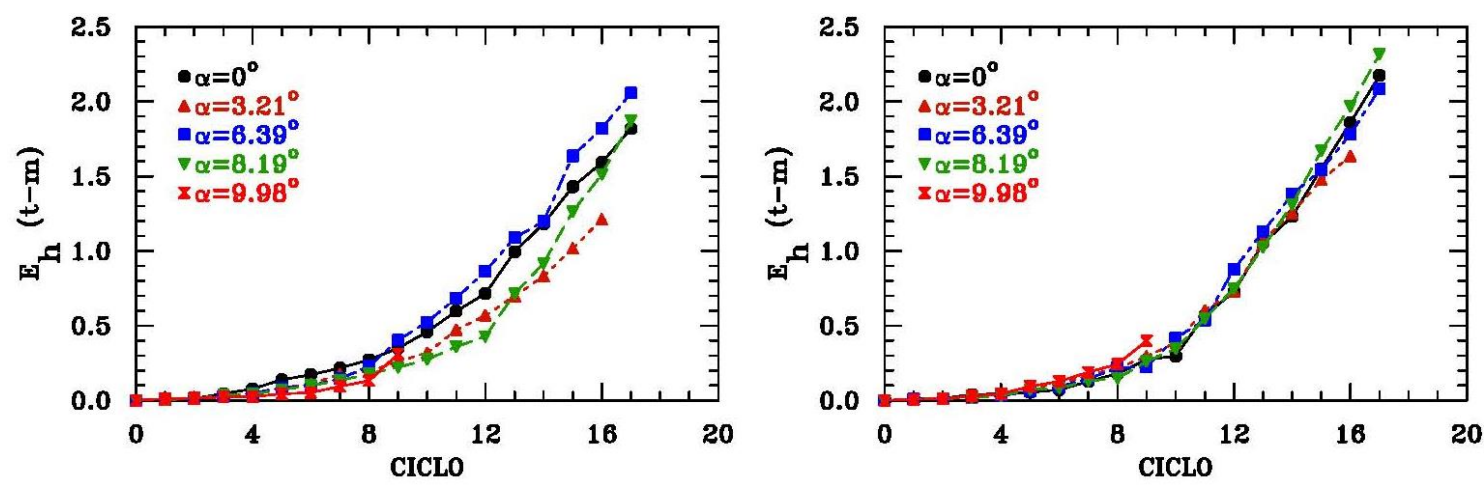

Figura 29. Energía histerética disipada acumulada, $E_{h}$, de los especímenes en estudio. Cartelas izquierdas y derechas en el lado izquierdo y derecho, respectivamente

Sin embargo, con la finalidad de hacer una comparación justa sobre la eficiencia de las trabes acarteladas en disipar energía, se evaluó la densidad de energía histerética acumulada $\left(U_{0}\right)$, definida como:

$$
U_{0} \cong \frac{\int_{0}^{\text {nciclos }} \partial E_{h}}{V_{\text {cartela }}}=\frac{E_{h}}{L_{h} b\left(\frac{h_{\text {max }}+h_{\text {min }}}{2}\right)}
$$

donde esencialmente se divide la energía histerética disipada acumulada (primeros y segundos ciclos) entre el volumen del elemento. En este caso, como las mediciones del cortante aplicado y la deformación medida involucra la extensión de la cartela $\left(L_{h}=125 \mathrm{~cm}\right)$, entonces en todos los casos se dividió la energía acumulada $\left(E_{h}\right)$ entre el volumen de la cartela (ecuación 11).

Las densidades de energía histerética acumuladas $U_{o}$ así determinadas para cada trabe y cartela se presentan en la figura 30. Se confirma básicamente lo reportado previamente en Archundia-Aranda et al. (2013) que, en general, a medida que aumenta el ángulo de acartelamiento $\alpha$, tiende a aumentar la densidad de energía histerética acumulada, $U_{0}$, lo cual se cumple al $100 \%$ en las cartelas derechas. Las excepciones en las cartelas izquierdas son el espécimen TASCV3 $\alpha 1-\mathrm{R} 1 \mathrm{c}\left(\alpha=3.21^{0}\right)$, el cual disipa menos energía que la trabe prismática $\left(\alpha=0^{0}\right)$, y el espécimen TASCV3 $\alpha 2-\mathrm{R} 1 \mathrm{c}\left(\alpha=6.39^{0}\right)$, que disipa más energía en prácticamente todos los ciclos que la trabe TASCV3 $\alpha 3-\mathrm{R} 1 \mathrm{c}\left(\alpha=8.19^{0}\right)$, aunque al colapso $U_{o}$ 
es muy similar. En el caso del espécimen que falló prematuramente (TASCV3 $\alpha 4-\mathrm{R} 1 \mathrm{c}, \alpha=9.98^{0}$ ), se aprecia que en los ciclos que duró el ensaye si existe una tendencia a disipar igual o mayor densidad de energía $U_{o}$ con respecto a los especímenes que finalmente disiparon mayor energía. Por lo tanto, sí se puede concluir que, en general, las cartelas son más eficientes para disipar energía por unidad de volumen que las trabes prismáticas, y que esta eficiencia es más evidente para ángulos $\alpha \geq 6^{0}$.
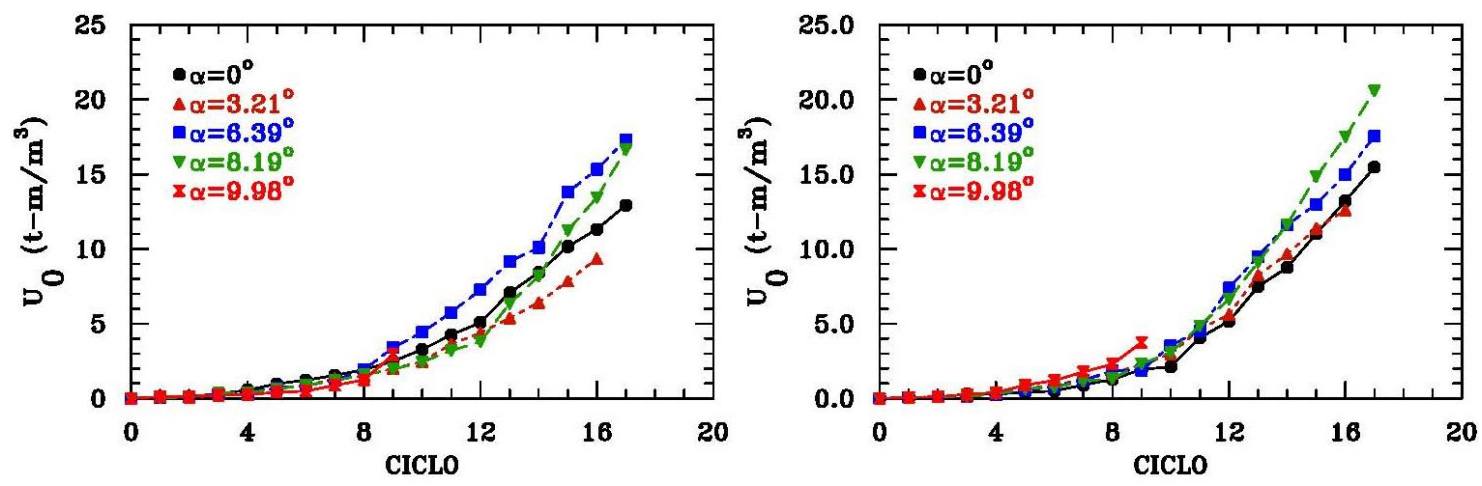

Figura 30. Densidad de energía histerética disipada acumulada, $U_{0}$, de los especímenes en estudio. Cartelas izquierdas y derechas en el lado izquierdo y derecho, respectivamente

\section{COMENTARIOS FINALES}

Se presentaron los resultados e interpretaciones de los ensayes de trabes acarteladas de concreto reforzado en condiciones de continuidad y sujetas a cargas cíclicas reversibles que permita estudiar su comportamiento a cortante. En este trabajo se reportan los resultados de las primeras cuatro trabes acarteladas y una prismática de concreto reforzado con refuerzo por cortante en la zona de las cartelas y que fueron diseñadas para fallar a cortante. Todos los especímenes tienen una longitud de acartelamiento de un tercio del claro libre y cubren uniformemente ángulos de acartelamiento entre cero (prismática) y diez grados. Los especímenes se sometieron a un patrón de cargas cíclicas reversibles y crecientes controladas por desplazamiento, donde los especímenes se sometieron a dos ciclos completos a la misma amplitud de desplazamiento, aumentando la amplitud de los ciclos de desplazamientos conforme a un patrón geométrico establecido.

Se examinaron las diferencias de comportamiento que presentan las trabes acarteladas con respecto a la prismática. Los resultados de los ensayes en condiciones de continuidad permiten corroborar lo observado en los ensayes anteriores en condiciones de apoyo simple: que las trabes acarteladas de concreto reforzado son más eficientes que las trabes prismáticas, aún cuando fallan por cortante. La afirmación anterior se sustenta en observar que, en general, en las trabes acarteladas: (1) se distribuye de mejor manera el agrietamiento, obteniéndose más grietas, pero de menor espesor o anchura que las pocas obtenidas en trabes prismáticas, (2) la degradación de rigidez disminuye a medida que aumenta el ángulo de acartelamiento, (3) la degradación de resistencia después de alcanzar la carga última $V_{u}$ es menos abrupta que en las trabes prismáticas y, (4) se disipa mayor energía por unidad de volumen (densidad de energía) a medida que aumenta el ángulo de acartelamiento. 


\section{AGRADECIMIENTOS}

Este trabajo formó parte del proyecto 79878 de Ciencia Básica de Conacyt intitulado Comportamiento cíclico a flexión y cortante de trabes acarteladas de concreto reforzado continuas, cuyo apoyo agradecen encarecidamente los autores. Agradecemos a la empresa HESA, en particular al Ing. José Antonio Sánchez Miravete, por asistirnos en la fabricación del acero de refuerzo (longitudinal y estribos), particularmente el inclinado. Durante la elaboración de los trabajos experimentales, intervinieron un gran número de personas, por lo que los autores agradecen a las siguientes personas de la UAM-A. Al maestro del laboratorio Rubén Barrera, Técnico José Luis Caballero, Técnico Juan Mateos, M. en I. Gilberto Rangel Torres y Sra. Silvia Ledesma por su apoyo institucional en el laboratorio. Al ingeniero Edznab López Rosas, quien participó y apoyó en estos experimentos como parte de su tesis de licenciatura y sus seminarios de tesis de maestría. Al ingeniero Jesús Iram Aranda Carrillo, que ensayó el espécimen TASCV $3 \alpha 4-R 1 c$ como parte de sus proyectos terminales y apoyó los experimentos como parte de sus talleres de ingeniería civil. El Ing. Eduardo Antonio Ayala Espejel apoyó a los experimentos como parte de su proyecto de integración y de sus talleres de ingeniería civil. El Ing. Luis Antonio Mendoza Valdez apoyó estos experimentos como parte de su proyecto terminal de licenciatura. El Ing. José Daniel Rivera Castro apoyó a los experimentos dentro de sus labores de ayudantía en el Área de Estructuras. Los ingenieros Ricardo Flores, Gadiel Martínez, Aldo Mendoza, Enrique Huerta, Juan Carlos Rodríguez, Mario Medina, Jovani Martínez, Sergio Reyes y Armando Colín, colaboraron tanto en sus talleres de ingeniería civil como en su servicio social. A todos los alumnos y alumnas que participaron en este proyecto como parte de sus talleres de ingeniería civil: Andrés Gama, Angel Liga, Alejandro Pérez, Gloria Saldívar, Nelly Orozco, Paola Martínez, Saraí Cisneros, Miriam Hernández, Carolina Viatela, Erik González, Irving Chávez, Erik Sánchez, Francisco Juárez, Fernando Arriaga, José Roldán, Juan Flores, Juan Sánchez, Julio Castaños, Oliver Narcizo, Saúl Rico, Carlos Morales, Jovani Cathi, Manuel López, Alex Cabrera, Abigail Velasco, Emmanuel Martínez, Jonathan Zendejas, Isaac Marín, Alan Herrera, Alan Hernández, Marco Islas, Jorge Rodríguez, Edgar Rojas, Erick Esquivel y Juan Antaño.

\section{REFERENCIAS}

ACI-318-14 (2014), "Building code requirements for structural concrete (ACI-318-14) and commentary (ACI 318R-14)", American Concrete Institute, Farmington Hills, Michigan, USA.

Aranda, J. I. (2015), "Estudio experimental del cortante en vigas acarteladas de concreto reforzado, con ángulos de $8^{\circ}$ y $10^{\circ}$ en cartela, construcción, instrumentación y demolición", Proyectos Terminales I y II, Departamento de Materiales, Universidad Autónoma Metropolitana, septiembre.

Archundia, H. I. (2004), "Comportamiento a cortante de trabes acarteladas de concreto reforzado ante carga estática”, Tesis de Maestría, División de Estudios de Posgrado de la Facultad de Ingeniería, Universidad Nacional Autónoma de México, mayo.

Archundia, H. I., A. Tena y O. M. González (2005), "Estudio experimental del cortante estático en trabes acarteladas de concreto reforzado", Reporte de Investigación 453, División de Ciencias Básicas e Ingeniería, Universidad Autónoma Metropolitana, junio, ISBN 970-31-0491-6.

Archundia, H. I., A. Tena y O. M. González (2006), "Mecanismos de resistencia y deformación a cortante de trabes acarteladas de concreto reforzado", Revista Internacional de Ingeniería de Estructuras, Vol. 11, No. 1, pp. 1-23.

Archundia, H. I., A. Tena, A. Grande y O. M. González (2007), "Comportamiento cíclico de trabes acarteladas de concreto reforzado sin refuerzo transversal que fallan por cortante", Revista de Ingeniería Sísmica, No. 76, pp. 89-112, enero-junio. DOI: https://doi.org/10.18867/ris-0. 
Archundia-Aranda, H. I. y A. Tena-Colunga (2008), "Cyclic behavior of reinforced concrete haunched beams failing in shear", Memorias, $14^{\text {th }}$ World Conference on Earthquake Engineering, Beijing, China, Artículo No. 12-01-0105, CD-ROM, octubre.

Archundia-Aranda, H. I., A. Tena-Colunga y A. Grande-Vega (2013), "Behavior of reinforced concrete haunched beams subjected to cyclic shear", Engineering Structures, Vol. 49, pp. 27-42, http://dx.doi.org/10.1016/j.engstruct.2012.10.037.

Archundia, H. I. (2013), "Recomendaciones de diseño a cortante de trabes acarteladas de concreto reforzado", Tesis de Doctorado, División de Estudios de Posgrado de la Facultad de Ingeniería, Universidad Nacional Autónoma de México, diciembre.

Archundia, H. I. y A. Tena (2015), "Diseño racional a cortante de trabes acarteladas de concreto reforzado", Concreto y Cemento. Investigación y Desarrollo, Vol. 6, No. 2, pp. 2-29, junio.

Debaiky, S.Y. y E. I. El-Niema (1982), "Behavior and strength of reinforced concrete haunched beams in shear”, ACI Journal, Vol. 79, No.3, pp. 184-194.

Dilger, W.H. y P. Langorh (1997), "Shear design of haunched concrete box girders of the Confederation Bridge", Canadian Journal of Civil Engineering, Vol. 24, No. 6, pp. 898-907. https://doi.org/10.1139/197-075.

El-Niema, E.I. (1988), "Investigation of concrete haunched beams under shear", ASCE Journal of Structural Engineering, Vol. 114, No. 4, pp. 917-930. https://doi.org/10.1061/(ASCE)07339445(1988)114:4(917).

Grande, A. (2005), "Comportamiento a cortante de trabes acarteladas de concreto reforzado sujetas a cargas cíclicas", Proyectos Terminales I y II, Departamento de Materiales, Universidad Autónoma Metropolitana, julio.

Grande, A. (2009), "Mecanismos de resistencia y deformación a cortante de trabes acarteladas de concreto reforzado sujetas a cargas cíclicas", Tesis de Maestría, Posgrado en Ingeniería Estructural, División de Ciencias Básicas e Ingeniería, Universidad Autónoma Metropolitana Azcapotzalco, julio.

Hou, C., K. Matsumoto y J. Niwa (2015), "Shear failure mechanism of reinforced concrete haunched beams", Journal of Japan Society of Civil Engineers, Vol. 3, No. 1, pp. 230-245. http://doi.org/10.2208/journalofjsce.3.1_230.

López, E. (2011), "Diseño de los especímenes, cimbra, instrumentación y revisión del dispositivo de prueba de las trabes acarteladas continuas a ensayarse cíclicamente en la UAM-A", Proyectos Terminales I y II, Departamento de Materiales, Universidad Autónoma Metropolitana, septiembre.

MacGregor, J. G. y J. K. Wight (2005), Reinforced concrete - mechanics and design, cuarta edición, Pearson-Prentice Hall, E.U.A.

MacLeod, I. A. y A. Houmsi (1994), "Shear strength of haunched beams without shear reinforcement", ACI Structural Journal, Vol. 91, No.1, pp. 79-89.

Marti, P. (1985), "Basic tools of reinforced concrete beam design", ACI Journal, Vol. 82, No. 1, pp. 4656.

Mörsch, E. (1952), Teoría y práctica del hormigón armado, Tomo II, Gili, Argentina.

Nghiep, V.-H. (2011), "Shear design of straight and haunched concrete beams without stirrups", Dissertation Doktor-Ingenieur (Tesis Doctoral), Technischen Universität Hamburg-Harburg, Hamburgo, Alemania.

Nielsen, M. P. (1999), Limit analysis and concrete plasticity, segunda edición, CRC Press, E.U.A. 
Nilson, A. H. (1999), Diseño de estructuras de concreto, duodécima edición, McGraw-Hill, Colombia.

NTCC-04 (2004), "Normas Técnicas Complementarias para Diseño y Construcción de Estructuras de Concreto", Gaceta Oficial del Distrito Federal, México, octubre.

Park R. y T. Paulay (1997), Estructuras de concreto reforzado, novena reimpresión de la primera edición, Limusa, México.

Pérez-Caldentey, A., P. Padilla, A. Muttoni y M. Fernández-Ruiz (2012), "Effect of load distribution and variable depth on shear resistance of slender beams without stirrups", ACI Structural Journal, Vol. 109, No. 5, pp. 595-604.

Stefanou, G. D. (1983), "Shear resistance for reinforced concrete beams with non-prismatic sections", Engineering Fracture Mechanics, Vol. 18, No. 3, pp. 643-667. https://doi.org/10.1016/00137944(83)90057-7.

Tena, A. (1993), "Recomendaciones para el detallado dúctil de trabes acarteladas de concreto reforzado ante sismos", Memorias, X Congreso Nacional de Ingeniería Sísmica, Puerto Vallarta, Jalisco, pp. 329-337, octubre.

Tena-Colunga, A. (1994), "Concerns regarding the seismic design of reinforced concrete haunched beams”, ACI Structural Journal, Vol. 90, No. 3, pp. 287-293, mayo-junio.

Tena-Colunga, A. (1996), "Stiffness formulation for nonprismatic beam elements", ASCE Journal of Structural Engineering, Vol. 122, No. 12, pp. 1484-1489, diciembre. http://dx.doi.org/10.1061/(ASCE)0733-9445(1996)122:4(439).

Tena-Colunga, A., H. I. Archundia-Aranda, A. Grande-Vega y O. M. González-Cuevas (2007), "Cyclic shear behavior of reinforced concrete haunched beams", Memorias, Ninth Canadian Conference on Earthquake Engineering (9CCEE), Ottawa, Canadá, CD-ROM, junio.

Tena-Colunga, A., H. I. Archundia-Aranda y O. M. González-Cuevas (2008), "Behavior of reinforced concrete haunched beams subjected to static shear loading", Engineering Structures, Vol. 30, No. 2, pp. 478-492. doi:10.1016/j.engstruct.2007.04.017.

Tena, A., L. A. Urbina, H. I. Archundia y E. López (2014), “Comportamiento cíclico a cortante de trabes acarteladas de concreto reforzado continuas", Memorias, XIX Congreso Nacional de Ingeniería Estructural, Puerto Vallarta, Jalisco, CDROM, Artículo 10-13, pp. 1-28, noviembre.

Tena, A., L. A. Urbina y H. I. Archundia (2017), "Trabes acarteladas de concreto reforzado continuas diseñadas para fallar por cortante. Parte 2: Mecanismo de resistencia a cortante", Revista de Ingeniería Sísmica, No. 97.

Urbina, L. A. (2013), "Comportamiento a cortante de trabes acarteladas continuas de concreto reforzado sujetas a cargas cíclicas", Tesis de Maestría, Posgrado en Ingeniería Estructural, División de Ciencias Básicas e Ingeniería, Universidad Autónoma Metropolitana Azcapotzalco, diciembre. 